\title{
Asymptotic Behavior of Densities for Stochastic Functional Differential Equations
}

\author{
Akihiro Kitagawa, ${ }^{1}$ and Atsushi Takeuchi ${ }^{2}$ \\ ${ }^{1}$ Aikou Educational Institute, Kinuyama 5-1610-1, Ehime Matsuyama, 791-8501, Japan \\ ${ }^{2}$ Department of Mathematics, Osaka City University, Sugimoto 3-3-138, Sumiyoshi-ku, \\ Osaka 558-8585, Japan \\ Correspondence should be addressed to Atsushi Takeuchi; takeuchi@sci.osaka-cu.ac.jp
}

Received 30 September 2012; Accepted 10 December 2012

Academic Editor: S. Mohammed

Copyright (C) 2013 A. Kitagawa and A. Takeuchi. This is an open access article distributed under the Creative Commons Attribution License, which permits unrestricted use, distribution, and reproduction in any medium, provided the original work is properly cited.

Consider stochastic functional differential equations depending on whole past histories in a finite time interval, which determine non-Markovian processes. Under the uniformly elliptic condition on the coefficients of the diffusion terms, the solution admits a smooth density with respect to the Lebesgue measure. In the present paper, we will study the large deviations for the family of the solution process and the asymptotic behaviors of the density. The Malliavin calculus plays a crucial role in our argument.

\section{Introduction}

Stochastic functional differential equations, or stochastic delay differential equations, determine non-Markovian processes, because the current states of the process in the equation depend on the past histories of the process. Such kind of equations was initiated by Itô and Nisio [1] in their pioneering work about 50 years ago. As stated in [2], there are some difficulties to study such equations, because we cannot use any methods in analysis, partial differential equations, and potential theory at all. On the other hand, it seems to be more natural to consider the models determined by the solutions to the stochastic functional differential equations in finance, physics, biology, and so forth, because such processes include their past histories and can be recognized to reflect real phenomena in various fields much more exactly.

The Malliavin calculus is well known as a powerful tool to study some properties on the density function by a probabilistic approach. There are a lot of works on the densities for diffusion processes by many authors, from the viewpoint of the Malliavin calculus (cf. [3]). Moreover it is also applicable to the case of solutions to stochastic functional differential equations, regarding as one of the examples of the Wiener functionals. Kusuoka and Stroock in [4] studied the application of the Malliavin calculus to the solutions to stochastic functional differential equations and obtained the result on the existence of the smooth density for the solution with respect to the Lebesgue measure. On the other hand, it is well known that the Malliavin calculus is very fruitful to study the asymptotic behavior of the density function related to the large deviations theory (cf. Léandre [5-8] and Nualart [9]). In fact, the Varadhan-type estimate of the density function for the diffusion processes can be also obtained from this viewpoint. Ferrante et al. in [10] discussed such problem in the case of stochastic delay differential equations, where the drift term depends on the whole past histories on the finite time interval, while the diffusion terms depend on the state only for the edges of the finite time interval. Mohammed and Zhang in [11] studied the large deviations for the solution process under a similar situation to [10]. But, the special forms on the diffusion terms play a crucial role throughout their arguments in $[10,11]$.

In the present paper, we will study the large deviations on the solution process to the stochastic functional differential equations. Our stochastic functional differential equations are much more general, because they are time inhomogeneous, and they are not only the drift terms, but also the diffusion terms in the equation depend on the whole past histories of the process over a finite interval. Furthermore, as a typical application of the large deviation theory and the Malliavin 
calculus, we will study the asymptotic behavior, so-called the Varadhan-type estimate, of the density function for the solution process, which is quite similar to the case of diffusion processes. The effect of the time delay plays a crucial role in the behavior of the density function, and the obtained result can be also regarded as the natural extension of the estimate for diffusion processes, which are the most interesting points in the present paper.

The paper is organized as follows. In Section 2, we will prepare some notations and introduce our stochastic functional differential equations. Section 3 will be devoted to the brief summary on the Malliavin calculus and its application to our equations. We will consider some estimates which guarantee the smoothness of the solution process and the non degeneracy in the Malliavin sense. The existence of the smooth density will be also discussed in Section 3. The negative-order moments of the Malliavin covariance matrix will be studied there which is important in order to give the estimate of the density function. Sections 4 and 5 are our main goals in the present paper. In Section 4, we will focus on the large deviation principles on the solution processes. As an application of the result obtained in Section 4, we will study the asymptotic behavior on the density for the solution process. Moreover, we can also derive the short time asymptotics on the density function, which can be interpreted as the generalization of the Varadhan-type estimate on diffusion processes (cf. [5-9]).

\section{Preliminaries}

Let $r$ and $T$ be positive constants, and denote an $m$-dimensional Brownian motion by $W=\left\{W(t)=\left(W^{1}(t), \ldots\right.\right.$, $\left.\left.W^{m}(t)\right) ; t \in[0, T]\right\}$. Let $A_{i}(i=0,1, \ldots, m)$ be $\mathbb{R}^{d}$-valued functions on $[0, T] \times C\left([-r, 0] ; \mathbb{R}^{d}\right)$ such that, for each $t \in$ $[0, T]$, the mapping $A_{i}(t, \cdot): C\left([-r, 0] ; \mathbb{R}^{d}\right) \quad \ni \quad f \mapsto$ $A_{i}(t, f) \in \mathbb{R}^{d}$ is smooth in the Frechét sense and all Frechét derivatives of any orders greater than 1 are bounded. Under the conditions stated above, the functions $A_{i}(i=0,1, \ldots, m)$ satisfy the linear growth condition and the Lipschitz condition in the functional sense of the form:

$$
\begin{gathered}
\sup _{t \in[0, T]} \sum_{i=0}^{m}\left|A_{i}(t, f)\right| \leq C_{1, T}\left(1+\|f\|_{\infty}\right), \\
\sup _{t \in[0, T]} \sum_{i=0}^{m}\left|A_{i}(t, f)-A_{i}(t, g)\right| \leq C_{2, T}\|f-g\|_{\infty},
\end{gathered}
$$

for $f, g \in C\left([-r, 0] ; \mathbb{R}^{d}\right)$, where $\|f\|_{\infty}=\sup _{t \in[-r, 0]}|f(t)|$. Denote by $A=\left(A_{1}, \ldots, A_{m}\right)$.

Let $0<\varepsilon \leq 1$ be sufficiently small. For a deterministic path $\eta \in C\left([-r, 0] ; \mathbb{R}^{d}\right)$, we will consider the $\mathbb{R}^{d}$-valued process $X^{\varepsilon}=\left\{X^{\varepsilon}(t) ; t \in[-r, T]\right\}$ given by the stochastic functional differential equation of the form:

$$
\begin{gathered}
X^{\varepsilon}(t)=\eta(t) \quad(t \in[-r, 0]), \\
d X^{\varepsilon}(t)=A_{0}\left(t, X_{t}^{\varepsilon}\right) d t+\varepsilon A\left(t, X_{t}^{\varepsilon}\right) d W(t) \quad(t \in(0, T]),
\end{gathered}
$$

where $X_{s}^{\varepsilon}=\left\{X^{\varepsilon}(s+u) ; u \in[-r, 0]\right\}$ is the segment. Since the current state of the solution depends on its past histories, the process $X^{\mathcal{E}}$ is non-Markovian clearly. Since the coefficients of (2) satisfy the Lipschitz and the linear growth condition in the functional sense, there exists a unique solution to (2), via the successive approximation $X^{\varepsilon,(n)}=\left\{X^{\varepsilon,(n)}(t) ; t \in[-r, T]\right\}$ $\left(n \in \mathbb{Z}_{+}\right)$of the solution process $X^{\varepsilon}$ to $(2)$ as follows:

$$
\begin{aligned}
X^{\varepsilon,(0)}(t) & =\eta(t) \quad(t \in[-r, 0]), \\
X^{\varepsilon,(0)}(t) & =\eta(0) \quad(t \in(0, T]), \\
X^{\mathcal{\varepsilon},(n)}(t) & =\eta(t) \quad(t \in[-r, 0]), \\
d X^{\varepsilon,(n)}(t) & =A_{0}\left(t, X_{t}^{\varepsilon,(n-1)}\right) d t \\
& +\varepsilon A\left(t, X_{t}^{\varepsilon,(n-1)}\right) d W(t) \quad(t \in(0, T]),
\end{aligned}
$$

for $n \in \mathbb{N}$ (cf. Itô and Nisio [1], Mohammed $[2,12])$.

Proposition 1. For any $p>1$, it holds that

$$
\sup _{0<\varepsilon \leq 1} \mathbb{E}\left[\sup _{t \in[-r, T]}\left|X^{\varepsilon}(t)\right|^{p}\right] \leq C_{3, p, T, \eta} .
$$

Proof. Let $p>2$ and $t \in[0, T]$. The Hölder inequality and the Burkholder inequality tell us to see that

$$
\begin{aligned}
\mathbb{E}\left[\sup _{\tau \in[-r, t]}\left|X^{\varepsilon}(\tau)\right|^{p}\right] \\
\leq C_{4, p}\|\eta\|_{\infty}^{p}+C_{4, p} \mathbb{E}\left[\sup _{\tau \in[0, t]}\left|X^{\varepsilon}(\tau)\right|^{p}\right] \\
\leq C_{4, p}\|\eta\|_{\infty}^{p}+C_{5, p} \mathbb{E}\left[\sup _{\tau \in[0, t]}\left|\int_{0}^{\tau} A_{0}\left(s, X_{s}^{\varepsilon}\right) d s\right|^{p}\right] \\
+C_{5, p} \varepsilon^{p} \mathbb{E}\left[\sup _{\tau \in[0, t]}\left|\int_{0}^{\tau} A\left(s, X_{s}^{\varepsilon}\right) d W(s)\right|^{p}\right] \\
\leq C_{4, p}\|\eta\|_{\infty}^{p}+C_{5, p} T^{p-1} \int_{0}^{t} \mathbb{E}\left[\left|A_{0}\left(s, X_{s}^{\varepsilon}\right)\right|^{p}\right] d s \\
+C_{6, p} \varepsilon^{p} T^{p / 2-1} \int_{0}^{t} \sum_{i=1}^{m} \mathbb{E}\left[\left|A_{i}\left(s, X_{s}^{\varepsilon}\right)\right|^{p}\right] d s \\
\leq C_{7, p, T, \eta}+C_{8, p, T} \int_{0}^{t} \mathbb{E}\left[\sup _{\tau \in[-r, s]}\left|X^{\varepsilon}(\tau)\right|^{p}\right] d s,
\end{aligned}
$$

from the linear growth condition on the coefficients $A_{i}(i=$ $0,1, \ldots, m)$. Hence, the Gronwall inequality enables us to obtain the assertion for $p>2$.

As for $1<p \leq 2$, the Jensen inequality yields us to see that

$$
\sup _{0<\varepsilon \leq 1} \mathbb{E}\left[\sup _{t \in[-r, T]}\left|X^{\varepsilon}(t)\right|^{p}\right] \leq\left(\sup _{0<\varepsilon \leq 1} \mathbb{E}\left[\sup _{t \in[-r, T]}\left|X^{\varepsilon}(t)\right|^{2 p}\right]\right)^{1 / 2},
$$

which implies the assertion by using the consequence stated above. The proof is complete. 


\section{Applications of the Malliavin Calculus}

At the beginning, we will introduce the outline of the Malliavin calculus on the Wiener space $C_{0}\left([0, T] ; \mathbb{R}^{m}\right)$, briefly, where $C_{0}\left([0, T] ; \mathbb{R}^{m}\right)$ is the set of $\mathbb{R}^{m}$-valued continuous functions on $[0, T]$ starting from the origin. See Di Nunno et al. [13] and Nualart $[9,14]$ for details. Let $H$ be the Cameron-Martin subspace of $C_{0}\left([0, T] ; \mathbb{R}^{m}\right)$ with the inner product

$$
\langle g, h\rangle_{H}=\int_{0}^{T} \dot{g}(t) \cdot \dot{h}(t) d t \quad(g, h \in H) .
$$

Denote by $\mathcal{S}$ the set of $\mathbb{R}$-valued random variables such that a random variable $F$ is represented as the following form:

$$
F(W)=f\left(W\left[h_{1}\right], \ldots, W\left[h_{n}\right]\right)
$$

for $W \in C_{0}\left([0, T] ; \mathbb{R}^{m}\right)$, where $h_{1}, \ldots, h_{n} \in H, W[h]=$ $\int_{0}^{T} h(s) \cdot d W(s)$ for $h \in H$, and $f \in C_{p}^{\infty}\left(\mathbb{R}^{n} ; \mathbb{R}\right)$. Here, we will denote by $C_{p}^{\infty}\left(\mathbb{R}^{n} ; \mathbb{R}\right)$ the set of smooth functions on $\mathbb{R}^{n}$ such that all derivatives of any orders have polynomial growth. For $k \in \mathbb{N}$, the $k$-times Malliavin-Shigekawa derivative $D^{k} F=$ $\left\{D_{u_{1}, \ldots, u_{k}}^{k} F ; u_{1}, \ldots, u_{k} \in[0, T]\right\}$ for $F \in \mathcal{S}$ is defined by

$$
D_{u_{1}, \ldots, u_{k}}^{k} F(W)=\left\{\begin{array}{cc}
\sum_{j=1}^{n} \partial_{j} f\left(W\left[h_{1}\right], \ldots, W\left[h_{n}\right]\right) & \\
\times \int_{0}^{u_{1}} h_{j}(s) d s & (k=1), \\
D_{u_{1}} \cdots D_{u_{k}} F(W) & (k \geq 2) .
\end{array}\right.
$$

We will consider $D^{0} F=F$, which helps us to define the operator $D^{k}$ for $k \in \mathbb{Z}_{+}$. For $p>1$ and $k \in \mathbb{Z}_{+}$, let $\mathbb{D}_{k, p}$ be the completion of $\mathcal{S}$ with respect to the norm

$$
\|F\|_{k, p}= \begin{cases}\left(\mathbb{E}\left[|F|^{p}\right]\right)^{1 / p} & (k=0), \\ \mathbb{E}\left[|F|^{p}\right]^{1 / p}+\sum_{j=1}^{k} \mathbb{E}\left[\left\|D^{j} F\right\|_{H^{\otimes j}}^{p}\right]^{1 / p} & (k \in \mathbb{N}) .\end{cases}
$$

Let $\mathbb{D}_{k, p}\left(\mathbb{R}^{d}\right)$ be the set of $\mathbb{R}^{d}$-valued random variables with the components of which belong to $\mathbb{D}_{k, p}$, and set $\mathbb{D}_{\infty}\left(\mathbb{R}^{d}\right)=$ $\bigcap_{p>1} \bigcap_{k \in \mathbb{Z}_{+}} \mathbb{D}_{k, p}\left(\mathbb{R}^{d}\right)$. For $F \in \mathbb{D}_{1,2}\left(\mathbb{R}^{d}\right)$, the $\mathbb{R}^{d} \otimes \mathbb{R}^{d}$-valued random variable $V_{F}$ given by

$$
V_{F}=\langle D F, D F\rangle_{H}=\int_{0}^{T} \frac{d}{d u} D_{u} F \cdot \frac{d}{d u} D_{u} F d u
$$

is well defined, which is called the Malliavin covariance matrix for $F$.

Before studying the application of the Malliavin calculus to the solution process $X^{\mathcal{E}}$ to (2), we will prepare two basic and well-known facts.

Lemma 2 (cf. Kusuoka and Stroock [4], Lemma 2.1). Let $\Gamma$ be a real separable Hilbert space, and $\alpha:[0, T] \times \Omega \rightarrow \mathbb{R}^{m} \otimes \Gamma$ be a progressively measurable process such that

$$
\mathbb{E}\left[\int_{0}^{T}\|\alpha(s)\|_{\mathbb{R}^{m} \otimes \Gamma}^{p} d s\right]<+\infty
$$

for all $p>1$. Then, for any $p>2$ and $\tau \in[0, T]$, it holds that

$$
\mathbb{E}\left[\sup _{t \in[0, \tau]}\left\|\int_{0}^{t} \alpha(s) d W(s)\right\|_{\Gamma}^{p}\right] \leq C_{9, p, \tau} \int_{0}^{\tau} \sum_{i=1}^{m} \mathbb{E}\left[\left\|\alpha_{i}(s)\right\|_{\Gamma}^{p}\right] d s .
$$

Lemma 3 (cf. Nualart [9], Proposition 1.3.8). Let $\{\beta(t) ; t \in$ $[0, T]\}$ be a $\left(\mathscr{F}_{t}\right)$-adapted, $\mathbb{R}^{m} \otimes \mathbb{R}^{d}$-valued process such that $\beta(t) \in \mathbb{D}_{1,2}\left(\mathbb{R}^{m} \otimes \mathbb{R}^{d}\right)$ for almost all $t \in[0, T]$, and that

$$
\mathbb{E}\left[\int_{0}^{T} \int_{0}^{T}\left|D_{u} \beta(t)\right|^{2} d u d t\right]<+\infty .
$$

Then, for each $t \in[0, T]$, it holds that $\int_{0}^{t} \beta(s) d W(s) \in$ $\mathbb{D}_{1,2}\left(\mathbb{R}^{d}\right)$, and that

$$
D_{u}\left(\int_{0}^{t} \beta(s) d W(s)\right)=\int_{0}^{u \wedge t} \beta(s) d s+\int_{0}^{t} D_{u} \beta(s) d W(s) .
$$

Now, we will return our position to study the application of the Malliavin calculus to the solution process $X^{\mathcal{\varepsilon}}$ to (2).

Proposition 4. Let $n \in \mathbb{Z}_{+}$and $0<\varepsilon \leq 1$. Then, for each $t \in$ $[-r, T]$, the $\mathbb{R}^{d}$-valued random variable $X^{\varepsilon,(n)}(t)$ is in $\mathbb{D}_{\infty}\left(\mathbb{R}^{d}\right)$. Moreover, for each $k \in \mathbb{Z}_{+}$, it holds that

$$
\begin{gathered}
\mathbb{E}\left[\sup _{t \in[-r, T]}\left\|D^{k} X^{\varepsilon,(n)}(t)\right\|_{H^{\otimes k} \otimes \mathbb{R}^{d}}^{p}\right] \leq C_{10, k, p, T, \eta}, \\
\mathbb{E}\left[\sup _{t \in[-r, T]}\left\|D^{k} X^{\varepsilon,(n)}(t)-D^{k} X^{\varepsilon,(n-1)}(t)\right\|_{H^{\otimes k} \otimes \mathbb{R}^{d}}^{p}\right] \leq \frac{C_{11, k, p, T, \eta}}{2^{n / 2}} .
\end{gathered}
$$

Proof. At the beginning, we will consider the case $p>2$ inductively on $k \in \mathbb{Z}_{+}$. As for $k=0$, it is a routine work to check the assertion via the Hölder inequality and the Burkholder inequality, from the Lipschitz condition and the linear growth condition on the coefficients $A_{i}(i=0,1, \ldots$, $m$ ), similarly to Proposition 1 . Next, we will discuss the case $k=1$. Let $n \in \mathbb{N}$, because the assertion for $n=0$ is trivial. Since $D X^{\varepsilon,(n)}=0$ for $t \in[-r, 0]$, we have only to prove the assertion for $t \in(0, T]$. The chain rule on the operator $D$ and Lemma 3 tell us to see that

$$
\begin{aligned}
D_{u} X^{\varepsilon,(n)}(t)= & \varepsilon \int_{0}^{u} A\left(s, X_{s}^{\varepsilon,(n-1)}\right) \rrbracket_{(s \leq t)} d s \\
& +\int_{0}^{t} \nabla A_{0}\left(s, X_{s}^{\varepsilon,(n-1)}\right) D_{u} X_{s}^{\mathcal{\varepsilon},(n-1)} d s \\
& +\varepsilon \int_{0}^{t} \nabla A\left(s, X_{s}^{\varepsilon,(n-1)}\right) D_{u} X_{s}^{\varepsilon,(n-1)} d W(s)
\end{aligned}
$$

for $u \in[0, T]$ (cf. Ferrante et al. [10], Lemma 6.1), where the symbol $\nabla$ is the Frechét derivative in $C\left([-r, 0] ; \mathbb{R}^{d}\right)$. Thus, the Hölder inequality and Lemma 2 enable us to get the assertions. Finally, we will discuss the general case $k \in \mathbb{Z}_{+}$. 
Suppose that the assertions are right until the case $n-1$. Remark that

$$
\begin{aligned}
D_{u_{1}, \ldots, u_{k}}^{k} & \left(\int_{0}^{t} A\left(s, X_{s}^{\varepsilon,(n-1)}\right) d W(s)\right) \\
= & D_{u_{1}, \ldots, u_{k-1}}^{k-1}\left(\int_{0}^{t} D_{u_{k}}\left(A\left(s, X_{s}^{\mathcal{\varepsilon},(n-1)}\right)\right) d W(s)\right) \\
& +D_{u_{1}, \ldots, u_{k-1}}^{k-1}\left(\int_{0}^{u_{k} \wedge t} A\left(s, X_{s}^{\varepsilon,(n-1)}\right) d s\right) \\
= & \ldots \\
= & \int_{0}^{t} D_{u_{1}, \ldots, u_{k}}^{k}\left(A\left(s, X_{s}^{\varepsilon,(n-1)}\right)\right) d W(s) \\
& +\sum_{\sigma \in \mathbb{S}_{k}} \int_{0}^{u_{\sigma(k)} \wedge t} D_{u_{\sigma(1)}^{k-1}, \ldots, u_{\sigma(k-1)}}^{k}\left(A\left(s, X_{s}^{\mathcal{\varepsilon},(n-1)}\right)\right) d s,
\end{aligned}
$$

from Lemma 3, where $\mathfrak{S}_{k}$ is the set of permutations of $\{1, \ldots, k\}$. Since

$$
\begin{aligned}
D_{u_{1}, \ldots, u_{k}}^{k} & \left(A_{i}\left(s, X_{s}^{\varepsilon,(n-1)}\right)\right) \\
= & D_{u_{1}, \ldots, u_{k-1}}^{k-1}\left(\nabla A_{i}\left(s, X_{s}^{\varepsilon,(n-1)}\right) D_{u_{k}} X_{s}^{\varepsilon,(n-1)}\right) \\
= & \sum_{\sigma \in \mathbb{S}_{k}} \sum_{j=0}^{k-1}\left(\begin{array}{c}
k-1 \\
j
\end{array}\right) D_{u_{\sigma(1)}, \ldots, u_{\sigma(k-j)}}^{k-1-j}\left(\nabla A_{i}\left(s, X_{s}^{\mathcal{\varepsilon},(n-1)}\right)\right) \\
& \times D_{u_{\sigma(k-j+1)}, \ldots, u_{\sigma(k-1)}, u_{k}}^{j+1} X_{s}^{\mathcal{\varepsilon},(n-1)}
\end{aligned}
$$

for $i=0,1, \ldots, m$, and

$$
\begin{aligned}
D_{u_{1}, \ldots, u_{k}}^{k} X^{\varepsilon,(n)}(t) & D_{u_{1}, \ldots, u_{k}}^{k}\left(\int_{0}^{t} A_{0}\left(s, X_{s}^{\varepsilon,(n-1)}\right) d s\right) \\
& +D_{u_{1}, \ldots, u_{k}}^{k}\left(\varepsilon \int_{0}^{t} A\left(s, X_{s}^{\mathcal{\varepsilon},(n-1)}\right) d W(s)\right) \\
= & \sum_{\sigma \in \mathfrak{S}_{k}} \varepsilon \int_{0}^{u_{\sigma(k)} \wedge t} D_{u_{\sigma(1)}, \ldots, u_{\sigma(k-1)}}^{k-1}\left(A\left(s, X_{s}^{\varepsilon,(n-1)}\right)\right) d s \\
& +\int_{0}^{t} D_{u_{1}, \ldots, u_{k}}^{k}\left(A_{0}\left(s, X_{s}^{\varepsilon,(n-1)}\right)\right) d s \\
& +\varepsilon \int_{0}^{t} D_{u_{1}, \ldots, u_{k}}^{k}\left(A\left(s, X_{s}^{\varepsilon,(n-1)}\right)\right) d W(s),
\end{aligned}
$$

we can get the assertion by using the Hölder inequality, Lemma 2 , and the assumption on the case until $k-1$ of the induction.

The case $1<p \leq 2$ is the direct consequence by the Jensen inequality. The proof is complete.

Proposition 5. For $t \in[-r, T]$, the $\mathbb{R}^{d}$-valued random variable $X^{\varepsilon}(t)$ is in $\mathbb{D}_{\infty}\left(\mathbb{R}^{m}\right)$. Moreover, for each $u \in[0, T]$, the
$\mathbb{R}^{m} \otimes \mathbb{R}^{d}$-valued process $\left\{D_{u} X^{\varepsilon}(t) ; t \in[-r, T]\right\}$ satisfies the equation of the form:

$$
\begin{aligned}
D_{u} X^{\varepsilon}(t)= & 0 \quad(t \in[-r, 0] \text { or } t<u) \\
D_{u} X^{\varepsilon}(t)= & \varepsilon \int_{0}^{u \wedge t} A\left(s, X_{s}^{\varepsilon}\right) d s+\int_{0}^{t} \nabla A_{0}\left(s, X_{s}^{\varepsilon}\right) D_{u} X_{s}^{\varepsilon} d s \\
& +\varepsilon \int_{0}^{t} \nabla A\left(s, X_{s}^{\varepsilon}\right) D_{u} X_{s}^{\varepsilon} d W(s) \quad(t \in[u, T]) .
\end{aligned}
$$

Proof. Let $p>1$ and $k \in \mathbb{Z}_{+}$be arbitrary. For each $t \in[-r, T]$, the sequence $\left\{X^{\varepsilon,(n)}(t) ; n \in \mathbb{N}\right\}$ is the Cauchy one in $\mathbb{D}_{k, p}\left(\mathbb{R}^{d}\right)$, from Proposition 4. Hence, we can find the limit, denoted by $\widetilde{X}^{\varepsilon}(t)$, in $\mathbb{D}_{k, p}\left(\mathbb{R}^{d}\right)$. Then, it is a routine work to see that the process $\left\{\widetilde{X}^{\varepsilon}(t) ; t \in[-r, T]\right\}$ satisfies (2), via the Hölder inequality and the Burkholder inequality, from the conditions on the coefficients $A_{i}(i=0,1, \ldots, m)$, which implies $\widetilde{X}^{\varepsilon}(t)=$ $X^{\varepsilon}(t)$ for $t \in[-r, T]$ from the uniqueness of the solutions. Thus, we can get $X(t) \in \mathbb{D}_{k, p}\left(\mathbb{R}^{d}\right)$ for $t \in[-r, T]$. Similarly, we can check that $\left\{D_{u} X(t) ; u \in[0, T]\right\}$ satisfies (21), by taking the limit in each term of (17) via the Hölder inequality and Lemma 2.

For $u \in[0, T]$, denote by $\left\{Z^{\varepsilon}(t, u) ; t \in[-r, T]\right\}$ the $\mathbb{R}^{d} \otimes$ $\mathbb{R}^{d}$-valued process determined by the following equation:

$$
\begin{aligned}
Z^{\varepsilon}(t, u)= & 0 \quad(t \in[-r, 0] \text { or } t<u) \\
Z^{\varepsilon}(u, u)= & I_{d} \\
d Z^{\varepsilon}(t, u)= & \nabla A_{0}\left(t, X_{t}^{\varepsilon}\right) Z_{t}^{\varepsilon}(\cdot, u) d t \\
& +\varepsilon \nabla A\left(t, X_{t}^{\varepsilon}\right) Z_{t}^{\varepsilon}(\cdot, u) d W(t) \quad(t \in(u, T])
\end{aligned}
$$

where $Z_{t}^{\varepsilon}(\cdot, u)=\left\{Z^{\varepsilon}(t+\tau, u) ; \tau \in[-r, 0]\right\}$.

\section{Corollary 6.}

$$
D_{u} X^{\varepsilon}(t)=\varepsilon \int_{0}^{u \wedge t} Z^{\varepsilon}(t, s) A\left(s, X_{s}^{\varepsilon}\right) d s .
$$

Proof. Direct consequence of Proposition 5 and the uniqueness of the solution to (21).

Finally, we will introduce the well-known criterion on the existence of the smooth density for the probability law of $X^{\varepsilon}(t)$ with respect to the Lebesgue measure on $\mathbb{R}^{d}$.

Lemma 7 (cf. Kusuoka and Stroock [4]). Suppose the uniformly elliptic condition on the coefficients $A_{i}(i=1, \ldots, m)$ of (2) as follows:

$$
\inf _{\zeta \in \mathbb{S}^{d-1}} \inf _{t \in[0, T]} \inf _{f \in C\left([-r, 0] ; \mathbb{R}^{d}\right)} \sum_{i=1}^{m}\left(\zeta \cdot A_{i}(t, f)\right)^{2}>0 .
$$

Then, for each $t \in(0, T]$ and $0<\varepsilon \leq 1$, there exists a smooth density $p^{\varepsilon}(t, y)$ for the probability law of $X^{\varepsilon}(t)$ with respect to the Lebesgue measure over $\mathbb{R}^{d}$. 
Proof. Since $X(t) \in \mathbb{D}_{\infty}\left(\mathbb{R}^{d}\right)$ from Proposition 5 , it is sufficiently to study that $\left(\operatorname{det} V^{\varepsilon}(t)\right)^{-1} \in \bigcap_{p>1} \mathbb{L}^{p}(\Omega)$ under the uniformly elliptic condition (24), where $V^{\varepsilon}(t)$ is the Malliavin covariance matrix for $X^{\varepsilon}(t)$. Denote by

$$
\widetilde{V}^{\varepsilon}(t)=\int_{0}^{t} \sum_{i=1}^{m} Z^{\varepsilon}(t, u) A_{i}\left(u, X_{u}^{\varepsilon}\right) A_{i}\left(u, X_{u}^{\varepsilon}\right)^{*} Z^{\varepsilon}(t, u)^{*} d u .
$$

Then, $V^{\varepsilon}(t)=\varepsilon^{2} \widetilde{V}^{\varepsilon}(t)$, so we have only to discuss the moment estimate on $\widetilde{V}^{\varepsilon}(t)$. As stated in Lemma 1 of Komatsu and Takeuchi [15], we will pay attention to the boundedness of

$$
\sup _{\zeta \in \mathbb{S}^{d-1}} \mathbb{E}\left[\left(\zeta \cdot \widetilde{V}^{\varepsilon}(t) \zeta\right)^{-p}\right]
$$

for any $p>1$, which is sufficient to our goal. Since

$$
\begin{aligned}
\mathbb{E}\left[\left(\zeta \cdot \widetilde{V}^{\mathcal{E}}(t) \zeta\right)^{-p}\right]= & \frac{1}{\Gamma(p)} \int_{0}^{+\infty} \lambda^{p-1} \\
& \times \mathbb{E}\left[\exp \left(-\lambda \zeta \cdot \widetilde{V}^{\varepsilon}(t) \zeta\right)\right] d \lambda
\end{aligned}
$$

we have to study the decay order of $\sup _{\zeta \in \mathbb{S}^{d-1}} \mathbb{E}[\exp (-\lambda \zeta$. $\left.\left.\widetilde{V}^{\varepsilon}(t) \zeta\right)\right]$ as $\lambda \rightarrow+\infty$.

Let $\lambda>1$ be sufficiently large. Remark that

$$
\mathbb{E}\left[\left\|Z^{\varepsilon}(t, u)-I_{d}\right\|_{\mathbb{R}^{d} \otimes \mathbb{R}^{d}}^{p}\right] \leq C_{12, p, T}(t-u)^{p / 2},
$$

for any $p>1$, from the Burkholder inequality and the Hölder inequality. Let $\xi>1 / 2,1<\gamma<2 \xi$ and $0<\sigma<(\gamma-1) / 2$. Write $t_{\xi}:=t-\lambda^{-\xi}$, and let $\zeta \in \mathbb{S}^{d-1}$. Then, we see that

$$
\begin{aligned}
\mathbb{E}\left[\exp \left(-\zeta \cdot \widetilde{V}^{\varepsilon}(t) \zeta\right)\right] \\
\leq \mathbb{E}_{1}\left[\exp \left(-\lambda \zeta \cdot \widetilde{V}^{\varepsilon}(t) \zeta\right)\right] \\
+\mathbb{P}\left[\int_{t_{\xi}}^{t}\left\|Z^{\varepsilon}(t, u)-I_{d}\right\|_{\mathbb{R}^{d} \otimes \mathbb{R}^{d}}^{2} d u \geq \lambda^{-\gamma}\right] \\
+\mathbb{P}\left[\sup _{s \in[-r, t]}\left|X^{\varepsilon}(s)\right| \geq \lambda^{\sigma}\right] \\
=: I_{1}+I_{2}+I_{3},
\end{aligned}
$$

where

$$
\begin{gathered}
\mathbb{E}_{1}[\cdot]:=\mathbb{E}\left[\cdot: \int_{t_{\xi}}^{t}\left\|Z^{\varepsilon}(t, u)-I_{d}\right\|_{\mathbb{R}^{d} \otimes \mathbb{R}^{d}}^{2} d u<\lambda^{-\gamma},\right. \\
\left.\sup _{s \in[-r, t]}\left|X^{\varepsilon}(s)\right|<\lambda^{\sigma}\right] .
\end{gathered}
$$

The Chebyshev inequality yields that

$$
\begin{aligned}
I_{2} & \leq \lambda^{\gamma p} \mathbb{E}\left[\left(\int_{t_{\xi}}^{t}\left\|Z^{\varepsilon}(t, u)-I_{d}\right\|_{\mathbb{R}^{d} \otimes \mathbb{R}^{d}}^{2} d u\right)^{p}\right] \\
& \leq C_{13, p, T} \lambda^{-(2 \xi-\gamma) p} .
\end{aligned}
$$

Similarly, the Chebyshev inequality leads to

$$
I_{3} \leq \lambda^{-\sigma p} \mathbb{E}\left[\sup _{s \in[-r, t]}\left|X^{\varepsilon}(s)\right|^{p}\right] \leq C_{14, p, T, \eta} \lambda^{-\sigma p}
$$

from Proposition 1. On the other hand, as for $I_{1}$, we have

$$
\begin{aligned}
I_{1} \leq & \mathbb{E}_{1}\left[\exp \left(-\lambda \int_{t_{\xi}}^{t} \sum_{i=1}^{m}\left|\zeta \cdot Z^{\varepsilon}(t, u) A_{i}\left(u, X_{u}^{\varepsilon}\right)\right|^{2} d u\right)\right] \\
\leq & \mathbb{E}_{1}\left[\exp \left(-\frac{\lambda}{2} \inf _{\zeta \in \mathbb{S}^{d-1}} \int_{t_{\xi}}^{t} \sum_{i=1}^{m}\left|\zeta \cdot A_{i}\left(u, X_{u}^{\varepsilon}\right)\right|^{2} d u\right)\right. \\
& \left.\quad \times \exp \left(\lambda \int_{t_{\xi}}^{t}\left\|Z^{\varepsilon}(t, u)-I_{d}\right\|_{\mathbb{R}^{d} \otimes \mathbb{R}^{d}}^{2} \sum_{i=1}^{m}\left|A_{i}\left(u, X_{u}^{\varepsilon}\right)\right|^{2} d u\right)\right] \\
\leq & \exp \left(\lambda^{1-\gamma+2 \sigma}\right) \\
& \times \exp \left(-\frac{\lambda}{2} \inf _{\zeta \in \mathbb{S}^{d-1}} \inf _{u \in[0, T]} \inf _{f \in C\left([-r, 0] ; \mathbb{R}^{d}\right.} \sum_{i=1}^{m}\left|\zeta \cdot A_{i}(u, f)\right|^{2}\right) \\
\leq & C_{15} \exp \left(-C_{16} \lambda\right) .
\end{aligned}
$$

Therefore, we can get

$$
\mathbb{E}\left[\exp \left(-\lambda \zeta \cdot \widetilde{V}^{\varepsilon}(t) \zeta\right)\right] \leq C_{17, p, T, \eta} \lambda^{-C_{18} p},
$$

so we have

$$
\begin{aligned}
\sup _{\zeta \in \mathbb{S}^{d-1}} \mathbb{E}\left[\left(\zeta \cdot V^{\varepsilon}(t) \zeta\right)^{-p}\right] & =\varepsilon^{-2 d p} \sup _{\zeta \in \mathbb{S}^{d-1}} \mathbb{E}\left[\left(\zeta \cdot \widetilde{V}^{\varepsilon}(t) \zeta\right)^{-p}\right] \\
& \leq C_{19, p, T} \varepsilon^{-2 d p}
\end{aligned}
$$

for any $p>1$. The proof is complete.

Remark 8. Consider the case

$$
A_{i}(t, f)=\widetilde{A}_{i}(t, f(0)) \quad(i=1, \ldots, m)
$$

where $\widetilde{A}_{i}:[0, T] \times \mathbb{R}^{d} \rightarrow \mathbb{R}^{d}$ with the good conditions on the boundedness and the regularity. Now, our stochastic functional differential equation is as follows:

$$
\begin{gathered}
X^{\varepsilon}(t)=\eta(t) \quad(t \in[-r, 0]), \\
d X^{\varepsilon}(t)=A_{0}\left(t, X_{t}^{\varepsilon}\right) d t+\varepsilon \widetilde{A}\left(t, X^{\varepsilon}(t)\right) d W(t) \quad(t \in(0, T]),
\end{gathered}
$$

where $\widetilde{A}=\left(\widetilde{A}_{1}, \ldots, \widetilde{A}_{m}\right)$. Then, we can get the same upper estimate of the inverse of the Malliavin covariance matrix $V^{\varepsilon}(t)$ for $X^{\varepsilon}(t)$ in the hypoelliptic situation, which means that the linear space generated by the vectors $\widetilde{A}_{i}(i=1, \ldots, m)$, and their Lie brackets span the space $\mathbb{R}^{d}$ (cf. Takeuchi [16]). 


\section{Large Deviation Principles for $X^{\varepsilon}$}

At the beginning, we will introduce the well-known fact on the sample-path large deviations for Brownian motions. See also [8]. Recall that $H$ is the Cameron-Martin space of $C_{0}\left([0, T] ; \mathbb{R}^{m}\right)$.

Lemma 9 (cf. Dembo and Zeitouni [17], Theorem 5.2.3). The family $\left\{\mathbb{P} \circ(\varepsilon W)^{-1} ; 0<\varepsilon \leq 1\right\}$ of the laws of $\varepsilon W$ over $C_{0}\left([0, T] ; \mathbb{R}^{m}\right)$ satisfies the large deviation principle with the good rate function $I$, where

$$
I(f)= \begin{cases}\frac{\|f\|_{H}^{2}}{2} & (f \in H), \\ +\infty & (f \notin H) .\end{cases}
$$

For $f \in H$, let $x^{f}=\left\{x^{f}(t) ; t \in[-r, T]\right\}$ be the solution to the following functional differential equation:

$$
\begin{gathered}
x^{f}(t)=\eta(t) \quad(t \in[-r, 0]) \\
d x^{f}(t)=A_{0}\left(t, x_{t}^{f}\right) d t+A\left(t, x_{t}^{f}\right) \dot{f}(t) d t \quad(t \in(0, T]) .
\end{gathered}
$$

Denote by

$$
\begin{aligned}
C_{\eta}\left([-r, T] ; \mathbb{R}^{d}\right)=\left\{w \in C\left([-r, T] ; \mathbb{R}^{d}\right) ;\right. \\
w(t)=\eta(t)(t \in[-r, 0])\} .
\end{aligned}
$$

Theorem 10. The family $\left\{\mathbb{P} \circ\left(X^{\varepsilon}\right)^{-1} ; 0<\varepsilon \leq 1\right\}$ of the laws of $X^{\varepsilon}$ over $C_{\eta}\left([-r, T] ; \mathbb{R}^{d}\right)$ satisfies the large deviation principle with the good rate function $\widetilde{I}$, where

$$
\widetilde{I}(g)=\inf \left\{I(f) ; f \in H, g=x^{f}\right\},
$$

and $I$ is the function given in Lemma 9.

Theorem 10 tells us to see, via the contraction principle (cf. Dembo and Zeitouni [17], Theorem 4.2.1).

Corollary 11. For each $t \in[0, T]$, the family $\left\{\mathbb{P} \circ\left(X^{\varepsilon}(t)\right)^{-1}\right.$; $0<\varepsilon \leq 1\}$ of the laws of $X^{\varepsilon}(t)$ over $\mathbb{R}^{d}$ satisfies the large deviation principle with the good rate function $\bar{I}$, where

$$
\bar{I}(y)=\inf \left\{\widetilde{I}(g) ; g \in C_{\eta}\left([-r, T] ; \mathbb{R}^{d}\right), y=g(t)\right\},
$$

and $\widetilde{I}$ is the function given in Theorem 10.

Now, we will prove Theorem 10, according to Azencott [18] and Léandre [5-8]. Our strategy stated here is almost parallel to $[10,11]$.

Proposition 12. For any $a>0$, the mapping

$$
H_{a}:=\left\{f \in H ;\|f\|_{H} \leq a\right\} \ni f \longmapsto x^{f} \in C_{\eta}\left([-r, T] ; \mathbb{R}^{d}\right)
$$

Proof. Let $f, g \in H_{a}$. Since

$$
x^{f}(t)=\eta(0)+\int_{0}^{t} A_{0}\left(s, x_{s}^{f}\right) d s+\int_{0}^{t} A\left(s, x_{s}^{f}\right) \dot{f}(s) d s,
$$

we see that

$$
\begin{aligned}
\sup _{\tau \in[-r, t]}\left|x^{f}(\tau)\right| \leq & \|\eta\|_{\infty}+\sup _{\tau \in[0, t]}\left|x^{f}(\tau)\right| \\
\leq & 2\|\eta\|_{\infty}+\int_{0}^{t}\left|A_{0}\left(s, x_{s}^{f}\right)\right| d s \\
& +\int_{0}^{t}\left\|A\left(s, x_{s}^{f}\right)\right\|_{\mathbb{R}^{m} \otimes \mathbb{R}^{d}}|\dot{f}(s)| d s \\
\leq & C_{20, T, \eta} \\
& +C_{21, T} \int_{0}^{t}(1+|\dot{f}(s)|) \\
& \times\left(1+\sup _{\tau \in[-r, s]}\left|x^{f}(\tau)\right|\right) d s,
\end{aligned}
$$

from the linear growth condition on $A_{i}(i=0,1, \ldots, m)$, which tells us to see that

$$
\sup _{\tau \in[-r, T]}\left|x^{f}(\tau)\right| \leq C_{22, T, \eta, a}
$$

On the other hand, since

$$
\begin{aligned}
x^{f}(t)-x^{g}(t)= & \int_{0}^{t}\left\{A_{0}\left(s, x_{s}^{f}\right)-A_{0}\left(s, x_{s}^{g}\right)\right\} d s \\
& +\int_{0}^{t}\left\{A\left(s, x_{s}^{f}\right) \dot{f}(s)-A\left(s, x_{s}^{g}\right) \dot{g}(s)\right\} d s,
\end{aligned}
$$

for $t \in(0, T]$, and the $\mathbb{R}^{d}$-valued functions $A_{i}(i=0,1, \ldots$, $m$ ) satisfy the Lipschitz condition and the linear growth condition, we have

$$
\begin{aligned}
\sup _{\tau \in[-r, t]} \mid & x^{f}(\tau)-x^{g}(\tau) \mid \\
= & \sup _{\tau \in[0, t]}\left|x^{f}(\tau)-x^{g}(\tau)\right| \\
\leq & \int_{0}^{t}\left|A_{0}\left(s, x_{s}^{f}\right)-A_{0}\left(s, x_{s}^{g}\right)\right| d s \\
& +\int_{0}^{t}\left\|A\left(s, x_{s}^{f}\right)-A\left(s, x_{s}^{g}\right)\right\|_{\mathbb{R}^{m} \otimes \mathbb{R}^{d}}|\dot{f}(s)| d s \\
& +\int_{0}^{t}\left\|A\left(s, x_{s}^{g}\right)\right\|_{\mathbb{R}^{m} \otimes \mathbb{R}^{d}}|\dot{f}(s)-\dot{g}(s)| d s \\
\leq & C_{23, T} \int_{0}^{t} \sup _{\tau \in[-r, s]}\left|x^{f}(\tau)-x^{g}(\tau)\right|\left(1+\sum_{i=1}^{m}\left|\dot{f}^{i}(s)\right|\right) d s \\
& +C_{24, T, \eta, a}\|f-g\|_{H \cdot}
\end{aligned}
$$

is continuous. 
The Gronwall inequality tells us to see that

$$
\begin{aligned}
\sup _{\tau \in[-r, t]} \mid & x^{f}(\tau)-x^{g}(\tau) \mid \\
\leq & C_{24, T, \eta, a}\|f-g\|_{H} \\
& \times \exp \left[C_{23, T} \int_{0}^{t}\left(1+\sum_{i=1}^{m}\left|\dot{f}^{i}(s)\right|\right) d s\right] \\
\leq & C_{25, T, \eta, a}\|f-g\|_{H},
\end{aligned}
$$

which completes the proof.

Proposition 13. Suppose that the $\mathbb{R}^{d}$-valued functions $A_{i}$ ( $i=$ $1, \ldots, m)$ are bounded. Then, for any $f \in H$ and $\rho>0$, there exist $\alpha_{\rho}>0$ and $\varepsilon_{\rho}>0$ such that

$$
\begin{gathered}
\mathbb{P}\left[\sup _{\tau \in[-r, T]}\left|X^{\varepsilon}(\tau)-x^{f}(\tau)\right|>\rho, \sup _{\tau \in[0, T]}|\varepsilon W(\tau)-f(\tau)| \leq \alpha_{\rho}\right] \\
\quad \leq C_{26, T, f, \rho} \exp \left[-C_{27, T, f} \frac{\rho^{2}}{\varepsilon^{2}}\right]
\end{gathered}
$$

for any $0<\varepsilon \leq \varepsilon_{\rho}$.

Proof. Define a new probability measure $d \widetilde{\mathbb{P}}$ by

$$
\left.\frac{d \widetilde{\mathbb{P}}}{d \mathbb{P}}\right|_{\mathscr{F}_{T}}=\exp \left[\int_{0}^{T} \sum_{i=1}^{m} \frac{\dot{f}^{i}(s)}{\varepsilon} d W^{i}(s)-\frac{\|f\|_{H}^{2}}{2 \varepsilon^{2}}\right]
$$

The Girsanov theorem tells us to see that the $\mathbb{R}^{m}$-valued process $\{\widetilde{W}(t):=W(t)-f(t) / \varepsilon ; t \in[0, T]\}$ is also the $m$ dimensional Brownian motion under the probability measure $d \widetilde{\mathbb{P}}$. Let $\left\{X^{\varepsilon, f}(t) ; t \in[-r, T]\right\}$ be the $\mathbb{R}^{d}$-valued process determined by the following equation:

$$
\begin{aligned}
X^{\varepsilon, f}(t)= & \eta(t) \quad(t \in[-r, 0]), \\
d X^{\varepsilon, f}(t)= & A_{0}\left(t, X_{t}^{\varepsilon, f}\right) d t \\
& +A\left(t, X_{t}^{\varepsilon, f}\right)\{\varepsilon d \widetilde{W}(t)+\dot{f}(t) d t\} \quad(t \in(0, T]) .
\end{aligned}
$$

Write $M(t):=\int_{0}^{t} A\left(s, X_{s}^{\varepsilon, f}\right) d \widetilde{W}(s)$. Remark that

$$
\begin{aligned}
\sup _{\tau \in[-r, t]}\left|X^{\varepsilon, f}(\tau)-x^{f}(\tau)\right| \\
=\sup _{\tau \in[0, t]}\left|X^{\varepsilon, f}(\tau)-x^{f}(\tau)\right|
\end{aligned}
$$

$$
\begin{aligned}
& \leq \int_{0}^{t}\left|A_{0}\left(s, X_{s}^{\varepsilon, f}\right)-A_{0}\left(s, x_{s}^{f}\right)\right| d s \\
& \quad+\int_{0}^{t}\left\|A\left(s, X_{s}^{\varepsilon, f}\right)-A\left(s, x_{s}^{f}\right)\right\|_{\mathbb{R}^{m} \otimes \mathbb{R}^{d}}|\dot{f}(s)| d s \\
& \quad+\sup _{\tau \in[0, t]}|\varepsilon M(\tau)| \\
& \leq C_{28, T} \int_{0}^{t} \sup _{\tau \in[-r, s]}\left|X^{\varepsilon, f}(\tau)-x^{f}(\tau)\right| \\
& \quad \times\left(1+\sum_{i=1}^{m}\left|\dot{f}^{i}(s)\right|\right) d s \\
& \quad+\sup _{\tau \in[0, t]}|\varepsilon M(\tau)| .
\end{aligned}
$$

The Gronwall inequality tells us to see that

$$
\begin{aligned}
\sup _{\tau \in[-r, t]}\left|X^{\varepsilon, f}(\tau)-x^{f}(\tau)\right| \\
\leq\left(\sup _{\tau \in[0, t]}|\varepsilon M(\tau)|\right) \\
\quad \times \exp \left[C_{28, T} \int_{0}^{t}\left(1+\sum_{i=1}^{m}\left|\dot{f}^{i}(s)\right|\right) d s\right] \\
\leq C_{29, T, f}\left(\sup _{\tau \in[0, t]}|\varepsilon M(\tau)|\right) .
\end{aligned}
$$

For each $k=1, \ldots, d$, the martingale representation theorem enables us to see that there exists a 1-dimensional Brownian motion $\left\{B^{k}(t) ; t \in[0, T]\right\}$ starting at the origin with

$$
\begin{gathered}
M^{k}(t)=B^{k}\left(\left\langle M^{k}\right\rangle(t)\right), \\
\left\langle M^{k}\right\rangle(t)=\int_{0}^{t} \sum_{i=1}^{m}\left|A_{i}^{k}\left(s, X_{s}^{\varepsilon, f}\right)\right|^{2} d s
\end{gathered}
$$

for $k=1, \ldots, d$. Remark that $\left\langle M^{k}\right\rangle(t) \leq C_{30, T}$, because of the boundedness of the $\mathbb{R}^{d}$-valued functions $A_{i}(i=1, \ldots, m)$. Since

$$
\begin{gathered}
\widetilde{\mathbb{P}}\left[\sup _{\tau \in\left[0, C_{30, T}\right]}\left|B^{k}(\tau)\right|>\frac{\rho}{C_{31, T, f} \mathcal{E}}\right] \\
\leq \sqrt{2} \exp \left[-\frac{\rho^{2}}{4 C_{30, T} C_{31, T, f}^{2} \mathcal{E}^{2}}\right],
\end{gathered}
$$


from the reflection principle on Brownian motions, we have

$$
\begin{aligned}
& \mathbb{P}\left[\sup _{\tau \in[-r, T]}\left|X^{\varepsilon}(\tau)-x^{f}(\tau)\right|>\rho, \sup _{\tau \in[0, T]}|\mathcal{E} W(\tau)-f(\tau)| \leq \alpha_{\rho}\right] \\
& =\widetilde{\mathbb{P}}\left[\sup _{\tau \in[-r, T]}\left|X^{\varepsilon, f}(\tau)-x^{f}(\tau)\right|>\rho, \sup _{\tau \in[0, T]}|\varepsilon \widetilde{W}(\tau)| \leq \alpha_{\rho}\right] \\
& \leq \widetilde{\mathbb{P}}\left[\sup _{\tau \in[0, T]}|M(\tau)|>\frac{\rho}{C_{29, T, f} \mathcal{E}}\right] \\
& \leq \widetilde{\mathbb{P}}\left[\sup _{\tau \in\left[0, C_{30, T}\right]}|B(\tau)|>\frac{\rho}{C_{29, T, f} \mathcal{E}}\right] \\
& \leq \widetilde{\mathbb{P}}\left[\bigcup_{k=1}^{d}\left\{\sup _{\tau \in\left[0, C_{30, T}\right]}\left|B^{k}(\tau)\right|>\frac{\rho}{C_{29, T, f} \sqrt{d} \varepsilon}\right\}\right] \\
& \leq \sqrt{2} d \exp \left[-\frac{\rho^{2}}{4 C_{30, T} C_{29, T, f}^{2} d \varepsilon^{2}}\right]
\end{aligned}
$$

which completes the proof.

\section{Proposition 14. It holds that}

$$
\lim _{R \rightarrow+\infty} \lim _{\varepsilon \succ 0} \sup \varepsilon \ln \mathbb{P}\left[\sup _{t \in[-r, T]}\left|X^{\varepsilon}(t)\right|>R\right]=-\infty
$$

Proof. Let $N>2$ be sufficient large. From the Itô formula, we see that

$$
\begin{aligned}
\left(1+\left|X^{\varepsilon}(t)\right|^{2}\right)^{N} & \\
= & \left(1+|\eta(0)|^{2}\right)^{N} \\
& +\int_{0}^{t} N\left(1+\left|X^{\varepsilon}(s)\right|^{2}\right)^{N-1} 2 \varepsilon X^{\varepsilon}(s) \cdot A\left(s, X_{s}^{\varepsilon}\right) d W(s) \\
+ & \int_{0}^{t}\left\{N\left(1+\left|X^{\varepsilon}(s)\right|^{2}\right)^{N-1}\right. \\
& \times\left(2 X^{\varepsilon}(s) \cdot A_{0}\left(s, X_{s}^{\varepsilon}\right)+\varepsilon^{2} \sum_{i=1}^{m}\left|A_{i}\left(s, X_{s}^{\varepsilon}\right)\right|^{2}\right) \\
& +2 N(N-1) \varepsilon^{2}\left(1+\left|X^{\varepsilon}(s)\right|^{2}\right)^{N-2} \\
& \left.\times \sum_{i=1}^{m}\left(X^{\varepsilon}(s) \cdot A_{i}\left(s, X_{s}^{\varepsilon}\right)\right)^{2}\right\} d s .
\end{aligned}
$$

Define $\sigma_{R}=\inf \left\{t>0 ;\left|X^{\varepsilon}(t)\right|>R\right\}$. Then, it holds that

$$
\begin{aligned}
& \mathbb{E}\left[\left(1+\left|X^{\varepsilon}\left(t \wedge \sigma_{R}\right)\right|^{2}\right)^{N}\right] \\
& \leq\left(1+\|\eta\|_{\infty}^{2}\right)^{N} \\
& +\mathbb{E}\left[\int _ { 0 } ^ { t \wedge \sigma _ { R } } \left\{N\left(1+\left|X^{\varepsilon}(s)\right|^{2}\right)^{N-1}\right.\right. \\
& \quad \times\left(2 X^{\varepsilon}(s) \cdot A_{0}\left(s, X_{s}^{\varepsilon}\right)+\varepsilon^{2} \sum_{i=1}^{m}\left|A_{i}\left(s, X_{s}^{\varepsilon}\right)\right|^{2}\right) \\
& +2 N(N-1) \varepsilon^{2}\left(1+\left|X^{\varepsilon}(s)\right|^{2}\right)^{N-2} \\
& \left.\left.\quad \times \sum_{i=1}^{m}\left(X^{\varepsilon}(s) \cdot A_{i}\left(s, X_{s}^{\varepsilon}\right)\right)^{2}\right\} d s\right] \\
& \leq\left(1+\|\eta\|_{\infty}\right)^{N}+C_{32, T}\left(N+\varepsilon^{2} N+\varepsilon^{2} N^{2}\right) \\
& \quad \times \mathbb{E}\left[\int_{0}^{t}\left(1+\left|X^{\varepsilon}\left(s \wedge \sigma_{R}\right)\right|^{2}\right)^{N} d s\right],
\end{aligned}
$$

from the linear growth condition on the coefficients $A_{i}(i=$ $0,1, \ldots, m)$ of $(2)$. Hence, the Gronwall inequality implies that

$$
\begin{aligned}
& \mathbb{E}\left[\left(1+\left|X^{\varepsilon}\left(t \wedge \sigma_{R}\right)\right|^{2}\right)^{N}\right] \\
& \quad \leq\left(1+\|\eta\|_{\infty}\right)^{N} \exp \left[C_{32, T}\left(N+\varepsilon^{2} N+\varepsilon^{2} N^{2}\right) t\right]
\end{aligned}
$$

In particular, taking $N=1 / \varepsilon$ yields that

$$
\begin{aligned}
\mathbb{E}[(1 & \left.\left.+\left|X^{\varepsilon}\left(t \wedge \sigma_{R}\right)\right|^{2}\right)^{1 / \varepsilon}\right] \\
& \leq\left(1+\|\eta\|_{\infty}\right)^{1 / \varepsilon} \exp \left[C_{33, T}\left(\frac{1}{\varepsilon}+1\right) t\right] .
\end{aligned}
$$

Therefore, the Chebyshev inequality leads us to see that

$$
\begin{aligned}
\mathbb{P}\left[\sup _{t \in[-r, T]}\left|X^{\varepsilon}(t)\right|>R\right] \\
\quad=\mathbb{P}\left[\sigma_{R} \leq T\right] \\
\quad \leq \mathbb{P}\left[\left|X^{\varepsilon}\left(T \wedge \sigma_{R}\right)\right| \geq R\right] \\
\quad \leq\left(1+R^{2}\right)^{-1 / \varepsilon} \mathbb{E}\left[\left(1+\left|X^{\varepsilon}\left(T \wedge \sigma_{R}\right)\right|^{2}\right)^{N}\right] \\
\quad \leq\left(\frac{1+\|\eta\|_{\infty}^{2}}{1+R^{2}}\right)^{1 / \varepsilon} \exp \left[C_{33, T}\left(\frac{1}{\varepsilon}+1\right) T\right]
\end{aligned}
$$


so we have

$$
\begin{gathered}
\underset{\varepsilon \downarrow 0}{\lim \sup } \varepsilon \ln \mathbb{P}\left[\sup _{t \in[-r, T]}\left|X^{\varepsilon}(t)\right|>R\right] \\
\quad \leq \ln \left(\frac{1+\|\eta\|_{\infty}^{2}}{1+R^{2}}\right)+C_{33, T} T
\end{gathered}
$$

which completes the proof.

Let $R \geq 1$. Define that $\sigma_{R}=\inf \left\{t>0 ;\left|X^{\mathcal{E}}(t)\right|>R\right\}$ and $X^{\varepsilon, R}(t)=X^{\varepsilon}\left(t \wedge \sigma_{R}\right)$

Proposition 15. For any $\delta>0$, it holds that

$$
\lim _{R \rightarrow+\infty} \lim _{\varepsilon \downarrow 0} \sup \varepsilon \ln \mathbb{P}\left[\sup _{t \in[-r, T]}\left|X^{\varepsilon}(t)-X^{\varepsilon, R}(t)\right|>\delta\right]=-\infty .
$$

Proof. Remark that

$$
\begin{aligned}
& \mathbb{P}\left[\sup _{t \in[-r, T]}\left|X^{\varepsilon}(t)-X^{\varepsilon, R}(t)\right|>\delta\right] \\
& \leq \mathbb{P}\left[\sup _{t \in[-r, T]}\left|X^{\varepsilon}(t)-X^{\varepsilon, R}(t)\right|>\delta, \sup _{t \in[-r, T]}\left|X^{\varepsilon}(t)\right| \leq R\right] \\
&+\mathbb{P}\left[\sup _{t \in[-r, T]}\left|X^{\varepsilon}(t)\right|>R\right] \\
&= \mathbb{P}\left[\sup _{t \in[-r, T]}\left|X^{\varepsilon}(t)-X^{\varepsilon, R}(t)\right|>\delta, \sigma_{R} \geq T\right] \\
&+\mathbb{P}\left[\sigma_{R} \leq T\right] \\
&= \mathbb{P}\left[\sigma_{R} \leq T\right] \\
& \leq \mathbb{P}\left[\left|X^{\varepsilon}\left(T \wedge \sigma_{R}\right)\right| \geq R\right] \\
& \leq\left(\frac{1+\|\eta\|_{\infty}^{2}}{1+R^{2}}\right)^{1 / \varepsilon} \exp \left[C_{33, T}\left(\frac{1}{\varepsilon}+1\right) T\right],
\end{aligned}
$$

as seen in the proof of Proposition 14. So, we can get

$$
\begin{gathered}
\limsup _{\varepsilon \downarrow 0} \varepsilon \ln \mathbb{P}\left[\sup _{t \in[-r, T]}\left|X^{\varepsilon}(t)-X^{\varepsilon, R}(t)\right|>\delta\right] \\
\quad \leq \ln \left(\frac{1+\|\eta\|_{\infty}^{2}}{1+R^{2}}\right)+C_{33, T} T,
\end{gathered}
$$

which completes the proof.

Proof of Theorem 10. We will prove the assertion in two steps of the form: the case where $A_{i}(i=1, \ldots, m)$ are bounded, and the general case on $A_{i}(i=1, \ldots, m)$.

Step 1. Suppose that the coefficients $A_{i}(i=1, \ldots, m)$ are bounded. Propositions 12 and 13 are sufficient to our goal (cf. $[17,18])$. In fact, the large deviation principle for the family
$\left\{\mathbb{P} \circ\left(X^{\varepsilon}\right)^{-1} ; 0<\varepsilon \leq 1\right\}$ comes from the one for $\left\{\mathbb{P} \circ(\varepsilon W)^{-1}\right.$; $0<\varepsilon \leq 1\}$ in Lemma 9 .

Step 2. We will discuss the general case on $A_{i}(i=1, \ldots, m)$. Let $R \geq 1$, and $F$ be a closed set in $C_{\eta}\left([-r, T] ; \mathbb{R}^{d}\right)$. Denote by $F_{R}=F \cap \overline{B(0 ; R)}$ and by $F_{R}^{\delta}$ the closed $\delta$-neighborhood of $F_{R}$, where $B(0 ; R)$ is the open ball in $C_{\eta}\left([-r, T] ; \mathbb{R}^{d}\right)$ with radius $R$ centered at $0 \in C_{\eta}\left([-r, T] ; \mathbb{R}^{d}\right)$. Then, it holds that

$$
\begin{aligned}
\mathbb{P}\left[X^{\varepsilon} \in F\right] \\
\leq \mathbb{P}\left[X^{\varepsilon} \in F, \sup _{t \in[-r, T]}\left|X^{\varepsilon}(t)\right| \leq R\right] \\
\quad+\mathbb{P}\left[\sup _{t \in[-r, T]}\left|X^{\varepsilon}(t)\right|>R\right] \\
=\mathbb{P}\left[X^{\varepsilon, R} \in F_{R}\right]+\mathbb{P}\left[\sup _{t \in[-r, T]}\left|X^{\varepsilon}(t)\right|>R\right] .
\end{aligned}
$$

As seen in Step 1, we have already obtained the large deviation principle for $\left\{\mathbb{P} \circ\left(X^{\varepsilon, R}\right)^{-1} ; 0<\varepsilon \leq 1\right\}$ with the good rate function $\widetilde{I}_{R}$, where $I(f)$ is given in Lemma 9 and

$$
\widetilde{I}_{R}(g)=\inf \left\{I(f) ; f \in H, g=x^{f}, \sup _{t \in[-r, T]}\left|x^{f}(t)\right| \leq R\right\} .
$$

So, we have

$$
\limsup _{\varepsilon \succ 0} \varepsilon \ln \mathbb{P}\left[X^{\varepsilon, R} \in F_{R}\right] \leq-\inf _{g \in F_{R}} \widetilde{I}_{R}(g) .
$$

Therefore, we can get

$$
\begin{aligned}
& \limsup _{\varepsilon \succ 0} \varepsilon \ln \mathbb{P}\left[X^{\varepsilon} \in F\right] \\
& \leq \lim _{R \rightarrow+\infty}\left\{\left(-\inf _{g \in F_{R}} \widetilde{I}_{R}(g)\right)\right. \\
& \left.\vee\left(\limsup _{\varepsilon \succ 0} \varepsilon \ln \mathbb{P}\left[\sup _{t \in[-r, T]}\left|X^{\varepsilon}(t)\right|>R\right]\right)\right\} \\
& =\lim _{R \rightarrow+\infty}\left(-\inf _{g \in F_{R}} \widetilde{I}(g)\right) \\
& \leq-\inf _{g \in F} \widetilde{I}(g),
\end{aligned}
$$

from Proposition 14, which completes the proof on the upper estimate of the large deviation principle.

Next, we will pay attention to the lower estimate of the large deviation principle. Let $G$ be an open set in 
$C_{\eta}\left([-r, T] ; \mathbb{R}^{d}\right)$, and take $\tilde{g}$ in $G \cap \overline{B(0 ; R)}$. Then, we can find $\delta>0$ such that $B(\tilde{g} ; \delta) \subset G$. Thus, we have

$$
\begin{aligned}
& -\widetilde{I}(\tilde{g})=-\widetilde{I}_{R}(\widetilde{g}) \\
& \leq-\inf _{g \in B(\tilde{g} ; \delta / 2)} \widetilde{I}_{R}(g) \\
& \leq \lim _{\varepsilon \downarrow 0} \inf \varepsilon \ln \mathbb{P}\left[X^{\varepsilon, R} \in B\left(\tilde{g} ; \frac{\delta}{2}\right)\right] \\
& \leq \lim _{\varepsilon \succ 0} \inf \varepsilon \\
& \times \ln \left\{\mathbb{P}\left[X^{\varepsilon} \in G\right]\right. \\
& \left.+\mathbb{P}\left[\sup _{t \in[-r, T]}\left|X^{\varepsilon}(t)-X^{\varepsilon, R}(t)\right|>\frac{\delta}{2}\right]\right\} \\
& \leq\left(\liminf _{\varepsilon \downarrow 0} \varepsilon \ln \mathbb{P}\left[X^{\varepsilon} \in G\right]\right) \\
& \vee\left(\liminf _{\varepsilon \unlhd 0} \varepsilon \ln \mathbb{P}\left[\sup _{t \in[-r, T]}\left|X^{\varepsilon}(t)-X^{\varepsilon, R}(t)\right|>\frac{\delta}{2}\right]\right) .
\end{aligned}
$$

The first equality is right, because of $\tilde{g} \in \overline{B(0 ; R)}$, while the third inequality is the consequence of the large deviation principle for $X^{\varepsilon, R}$ as seen in Step 1. The forth inequality is right, because $X^{\varepsilon} \in B(\tilde{g} ; \delta / 2)^{c}$ under $X^{\varepsilon, R} \in B(\tilde{g} ; \delta)^{c}$ and $\sup _{t \in[-r, T]}\left|X^{\varepsilon}(t)-X^{\varepsilon, R}(t)\right| \leq \delta / 2$. Taking the limit as $R \rightarrow$ $+\infty$ leads us to see that

$$
-\widetilde{I}(\widetilde{g}) \leq \lim _{\varepsilon \searrow 0} \inf \varepsilon \ln \mathbb{P}\left[X^{\varepsilon} \in G\right],
$$

from Proposition 15, which completes the proof on the lower estimate of the large deviation principle. The proof of Theorem 10 is complete.

\section{Density Estimates}

In this section, we will consider the estimate of the density $p^{\varepsilon}(t, y)$ for the solution $X^{\varepsilon}(t)$, from the viewpoint of the Malliavin calculus.

Theorem 16 (Upper estimate). Suppose that the $\mathbb{R}^{d}$-valued functions $A_{i}(i=1, \ldots, m)$ satisfy the uniformly elliptic condition (24). Then, it holds that

$$
\lim _{\varepsilon \succ 0} \sup \varepsilon^{2} \ln p^{\varepsilon}(t, y) \leq-\bar{I}(y),
$$

where the function $\bar{I}$ is given in Corollary 11.

Proof. Let $0<\sigma<1$ be sufficiently small, and $\Lambda_{\sigma} \epsilon$ $C_{0}^{\infty}\left(\mathbb{R}^{d} ;[0,1]\right)$ such that

$$
\Lambda_{\sigma}(z)= \begin{cases}1 & (|z-y| \leq \sigma) \\ 0 & (|z-y|>2 \sigma)\end{cases}
$$

Take $U=\prod_{j=1}^{d}\left[a_{j}, b_{j}\right] \subset \mathbb{R}^{d}$ such that $U \subset \operatorname{Supp}\left[\Lambda_{\sigma}\right]$. Then, the integration by parts formula tells us to see that

$$
\begin{aligned}
& \mathbb{P}\left[X^{\varepsilon}(t) \in U\right] \\
& =\mathbb{E}\left[\mathbb{q}_{U}\left(X^{\varepsilon}(t)\right) \Lambda_{\sigma}\left(X^{\varepsilon}(t)\right)\right] \\
& =\mathbb{E}\left[\int_{-\infty}^{X^{\varepsilon, 1}(t)} \ldots \int_{-\infty}^{X^{\varepsilon, d}(t)} \mathbb{q}_{U}\left(y_{1}, \ldots, y_{d}\right) d y_{1}\right. \\
& \left.\ldots d y_{d} \Gamma_{(1, \ldots, d)}\left(X^{\varepsilon}(t), \Lambda_{\sigma}\left(X^{\varepsilon}(t)\right)\right)\right] \\
& =\int_{U}\left[\prod_{j=1}^{d} \mathbb{q}_{\left(y_{j},+\infty\right)}\left(X^{\varepsilon, j}(t)\right)\right. \\
& \left.\times \Gamma_{(1, \ldots, d)}\left(X^{\varepsilon}(t), \Lambda_{\sigma}\left(X^{\varepsilon}(t)\right)\right)\right] d y_{1} \ldots d y_{d},
\end{aligned}
$$

where

$$
\begin{aligned}
& \Gamma_{j}\left(X^{\varepsilon}(t), \Lambda_{\sigma}\left(X^{\varepsilon}(t)\right)\right) \\
& =\delta\left(\Lambda_{\sigma}\left(X^{\varepsilon}(t)\right) \sum_{k=1}^{d}\left[\left(V^{\varepsilon}(t)\right)^{-1}\right]_{j k} D X^{\varepsilon, k}(t)\right), \\
& \Gamma_{(1, \ldots, d)}\left(X^{\varepsilon}(t), \Lambda_{\sigma}\left(X^{\varepsilon}(t)\right)\right) \\
& =\Gamma_{d}\left(X^{\varepsilon}(t), \Gamma_{(1, \ldots, d-1)}\left(X^{\varepsilon}(t), \Lambda_{\sigma}\left(X^{\varepsilon}(t)\right)\right)\right),
\end{aligned}
$$

and $\delta$ is the Skorokhod integral operator. Remark that, under the uniformly elliptic condition (24) on the $\mathbb{R}^{d}$-valued functions $A_{i}(i=1, \ldots, m)$,

$$
\begin{aligned}
& \left\|\Gamma_{(1, \ldots, d)}\left(X^{\mathcal{\varepsilon}}(t), \Lambda_{\sigma}\left(X^{\mathcal{\varepsilon}}(t)\right)\right)\right\|_{\mathbb{L}^{p}(\Omega)} \\
& \quad \leq C_{34, p, T, \eta}\left\|\left(V^{\varepsilon}(t)\right)^{-1}\right\|_{\mathbb{L}^{\alpha}(\Omega)}\left\|X^{\varepsilon}(t)\right\|_{\beta, \gamma}\left\|\Lambda_{\sigma}\left(X^{\varepsilon}(t)\right)\right\|_{\kappa, \sigma} \\
& \quad \leq C_{35, p, T, \eta} \varepsilon^{-2 d},
\end{aligned}
$$

where $\alpha, \gamma, \sigma>1$ and $\beta, \kappa \in \mathbb{Z}_{+}$, by using Proposition 5 and the proof of Lemma 7. Hence, the density $p^{\varepsilon}(t, y)$ can be estimated from the above as follows:

$$
\begin{aligned}
& p^{\varepsilon}(t, y) \\
& \quad=\mathbb{E}\left[\prod_{j=1}^{d} \rrbracket_{\left(y_{j},+\infty\right)}\left(X^{\varepsilon, j}(t)\right) \Gamma_{(1, \ldots, d)}\left(X^{\varepsilon}(t), \Lambda_{\sigma}\left(X^{\varepsilon}(t)\right)\right)\right] \\
& \quad \leq \mathbb{E}\left[\left|\Gamma_{(1, \ldots, d)}\left(X^{\varepsilon}(t), \Lambda_{\sigma}\left(X^{\varepsilon}(t)\right)\right)\right| \square_{\operatorname{Supp}\left[\Lambda_{\sigma}\right]}\left(X^{\varepsilon}(t)\right)\right] \\
& \quad \leq C_{35, p, T, \eta} \varepsilon^{-2 d} \mathbb{P}\left[X^{\varepsilon}(t) \in \operatorname{Supp}\left[\Lambda_{\sigma}\right]\right]^{1 / q},
\end{aligned}
$$


where $q>1$ such that $1 / p+1 / q=1$. From Corollary 11 , we have

$$
\limsup _{\varepsilon \searrow 0} \varepsilon^{2} \ln \mathbb{P}\left[X^{\varepsilon}(t) \in \operatorname{Supp}\left[\Lambda_{\sigma}\right]\right] \leq-\inf _{z \in \operatorname{Supp}\left[\Lambda_{\sigma}\right]} \bar{I}(z) .
$$

Since the function $\bar{I}$ is a lower semicontinuous, taking the limit as $\sigma \searrow 0$ and $q \searrow 1$ enables us to see that

$$
\limsup _{\varepsilon \searrow 0} \varepsilon^{2} \ln p^{\varepsilon}(t, y) \leq-\bar{I}(y),
$$

which is the conclusion of Theorem 16.

Remark 17. As stated in Remark 8, a similar problem can be also studied under the hypoelliptic condition, in the case

$$
A_{i}(t, f)=\widetilde{A}_{i}(t, f(0)) \quad(i=1, \ldots, m),
$$

where $\widetilde{A}_{i}:[0, T] \times \mathbb{R}^{d} \rightarrow \mathbb{R}^{d}$ with the good conditions on the boundedness and the regularity (cf. [16]).

Now, we will study the lower estimate of the density $p^{\varepsilon}(t, y)$ for the solution process $X^{\varepsilon}$ to (2). Before doing it, we will prepare some arguments.

Proposition 18. Let $f \in H$, and assume the uniformly elliptic condition (24) on the functions $A_{i}(i=1, \ldots, m)$. Then, it holds that

$$
\operatorname{det} v^{f}(t)>0,
$$

for each $t \in(0, T]$, where $v^{f}(t)$ is the Gram matrix for $x^{f}(t)$.

Proof. Let $u \in[0, T]$, and $\{\bar{Z}(t, u) ; t \in[-r, T]\}$ be the $\mathbb{R}^{d} \otimes \mathbb{R}^{d}$-valued mappings given by the following functional differential equation:

$$
\begin{aligned}
\bar{Z}(t, u)= & 0 \quad(t \in[-r, 0] \text { or } t \in(0, u)), \\
d \bar{Z}(t, u)= & \nabla A_{0}\left(t, x_{t}^{f}\right) \bar{Z}_{t}(\cdot, u) d t \\
& +\nabla A\left(t, x_{t}^{f}\right) \bar{Z}_{t}(\cdot, u) \dot{f}(t) d t \quad(t \in[u, T]),
\end{aligned}
$$

where $\bar{Z}_{t}(\cdot, u)=\{\bar{Z}(t+\tau, u) ; \tau \in[-r, 0]\}$. From the condition on the coefficients $A_{i}(i=0,1, \ldots, m)$, we see that

$$
\begin{aligned}
\sup _{\tau \in[u, t]}\left\|\bar{Z}(\tau, u)-I_{d}\right\|_{\mathbb{R}^{d} \otimes \mathbb{R}^{d}} & \\
\leq & \sup _{\tau \in[u, t]}\left\|\int_{\mathcal{u}}^{\tau} \nabla A_{0}\left(s, x_{s}^{f}\right) \bar{Z}_{s}(\cdot, u) d s\right\|_{\mathbb{R}^{d} \otimes \mathbb{R}^{d}} \\
& \quad+\sup _{\tau \in[u, t]}\left\|\int_{\mathcal{u}}^{\tau} \nabla A\left(s, x_{s}^{f}\right) \bar{Z}_{s}(\cdot, u) \dot{f}(s) d s\right\|_{\mathbb{R}^{d} \otimes \mathbb{R}^{d}}
\end{aligned}
$$

$$
\begin{aligned}
& \leq \int_{u}^{t}\left(\left\|\nabla A_{0}\left(s, x_{s}^{f}\right)\right\|_{C\left([-r, 0] ; \mathbb{R}^{d}\right) \otimes \mathbb{R}^{d}}\right. \\
& \left.\quad+\left\|\nabla A\left(s, x_{s}^{f}\right)\right\|_{C\left([-r, 0] ; \mathbb{R}^{d}\right) \otimes \mathbb{R}^{m} \otimes \mathbb{R}^{d}}|\dot{f}(s)|\right) d s \\
& \quad+\int_{\mathcal{u}}^{t}\left(\left\|\nabla A_{0}\left(s, x_{s}^{f}\right)\right\|_{C\left([-r, 0] ; \mathbb{R}^{d}\right) \otimes \mathbb{R}^{d}}\right. \\
& \left.\quad+\left\|\nabla A\left(s, x_{s}^{f}\right)\right\|_{C\left([-r, 0] ; \mathbb{R}^{d}\right) \otimes \mathbb{R}^{m} \otimes \mathbb{R}^{d}}|\dot{f}(s)|\right) \\
& \quad \sup _{\tau \in[s-r, s]}\left\|\bar{Z}(\tau, u)-I_{d}\right\|_{\mathbb{R}^{d} \otimes \mathbb{R}^{d}} d s \\
& \leq C_{36, T} \int_{u}^{t}\left(1+\sum_{i=1}^{m}\left|\dot{f}^{i}(s)\right|\right) d s \\
& \quad+C_{37, T} \int_{u}^{t}\left(1+\sum_{i=1}^{m}\left|\dot{f}^{i}(s)\right|\right) \\
& \quad \times \sup _{\tau \in[u, s]}\left\|\bar{Z}(\tau, u)-I_{d}\right\|_{\mathbb{R}^{d} \otimes \mathbb{R}^{d}} d s .
\end{aligned}
$$

Remark that

$$
\int_{u}^{t}\left(1+\sum_{i=1}^{m}\left|\dot{f}^{i}(s)\right|\right) d s \leq C_{38, f, T}(t-u)^{1 / 2} .
$$

Hence, the Gronwall inequality tells us to see that

$$
\sup _{\tau \in[u, t]}\left\|\bar{Z}(\tau, u)-I_{d}\right\|_{\mathbb{R}^{d} \otimes \mathbb{R}^{d}} \leq C_{39, f, T}(t-u)^{1 / 2} .
$$

On the other hand, remark that we have already seen in the proof of Proposition 12 that

$$
\sup _{\tau \in[-r, t]}\left|x^{f}(\tau)\right| \leq C_{22, T, \eta, f} .
$$

Now, we will pay attention to the lower estimate of $\operatorname{det} v^{f}(t)$. Since, for each $u \in[0, T],\left\{D_{u} x^{f}(t) ; t \in[-r, T]\right\}$ satisfies the equation

$$
\begin{aligned}
D_{u} x^{f}(t)= & 0 \quad(t \in[-r, 0]), \\
D_{u} x^{f}(t)= & \int_{0}^{u} A\left(s, x_{s}^{f}\right) \mathbb{\square}_{(s \leq t)} d s+\int_{0}^{t} \nabla A_{0}\left(s, x_{s}^{f}\right) D_{u} x_{s}^{f} d s \\
& +\int_{0}^{t} \nabla A\left(s, x_{s}^{f}\right) D_{u} x_{s}^{f} \dot{f}(s) d s \quad(t \in(0, T]),
\end{aligned}
$$

we have

$$
D_{u} x^{f}(t)=\int_{0}^{u \wedge t} \bar{Z}(t, s) A\left(s, x_{s}^{f}\right) d s,
$$

similarly to Corollary 6 . Hence, the Gram matrix $v^{f}(t)$ can be expressed as follows:

$$
v^{f}(t)=\int_{0}^{t} \bar{Z}(t, u) A\left(u, x_{u}^{f}\right) A\left(u, x_{u}^{f}\right)^{*} \bar{Z}(t, u)^{*} d u .
$$


Let $t_{\alpha} \in[0, T]$ be sufficiently close to $t$. So, we see that

$$
\begin{aligned}
& \operatorname{det} v^{f}(t) \\
& =\operatorname{det}\left[\int_{0}^{t} \bar{Z}(t, u) A\left(u, x_{u}^{f}\right) A\left(u, x_{u}^{f}\right)^{*} \bar{Z}(t, u)^{*} d u\right] \\
& \geq\left\{\inf _{\zeta \in \mathbb{S}^{d-1}} \int_{0}^{t} \sum_{i=1}^{m}\left(\zeta \cdot \bar{Z}(t, u) A_{i}\left(u, x_{u}^{f}\right)\right)^{2} d u\right\}^{d} \\
& =\left\{\inf _{\zeta \in \mathbb{S}^{d-1}} \int_{0}^{t} \sum_{i=1}^{m}\left(\zeta \cdot \bar{Z}(t, u) A_{i}\left(u, x_{u}^{f}\right)\right)^{2} d u\right\}^{d} \\
& \times \llbracket\left(\sup _{t \in[-r, T]}\left|x^{f}(t)\right| \leq C_{22, T, \eta, f}\right) \\
& \times \mathbb{}\left(\sup _{u \in\left[t_{\alpha}, t\right]}\left\|\bar{Z}(t, u)-I_{d}\right\|_{\mathbb{R}^{d} \otimes \mathbb{R}^{d}} \leq C_{39, T, f}\left(t-t_{\alpha}\right)^{1 / 2}\right) \\
& \geq\left\{\frac{1}{2} \inf _{\zeta \in \mathbb{S}^{d-1}} \int_{t_{\alpha}}^{t} \sum_{i=1}^{m}\left(\zeta \cdot A_{i}\left(u, x_{u}^{f}\right)\right)^{2} d u\right. \\
& \left.-\int_{t_{\alpha}}^{t} \sum_{i=1}^{m}\left\|\bar{Z}(t, u)-I_{d}\right\|_{\mathbb{R}^{d} \otimes \mathbb{R}^{d}}^{2}\left|A_{i}\left(u, x_{u}^{f}\right)\right|^{2} d u\right\}^{d} \\
& \times \square\left(\sup _{t \in[-r, T]}\left|x^{f}(t)\right| \leq C_{22, T, \eta, f}\right)
\end{aligned}
$$

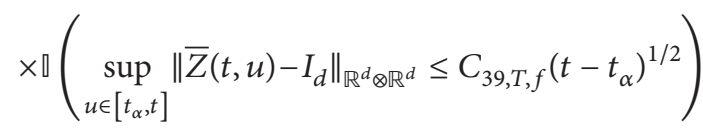

$$
\begin{aligned}
& \geq\left\{\frac{t-t_{\alpha}}{2} \inf _{\zeta, t, g} \sum_{i=1}^{m}\left(\zeta \cdot A_{i}(t, g)\right)^{2}-C_{40, T, \eta, f}\left(t-t_{\alpha}\right)^{2}\right\}^{d} \\
& \times \llbracket\left(\sup _{t \in[-r, T]}\left|x^{f}(t)\right| \leq C_{22, T, \eta, f}\right)
\end{aligned}
$$

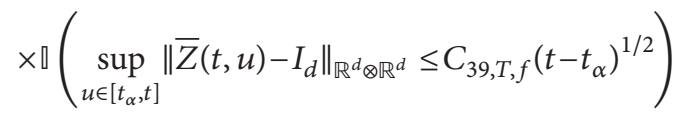

$$
\begin{aligned}
& \geq C_{41, T, \eta, f}\left(t-t_{\alpha}\right)^{d}
\end{aligned}
$$

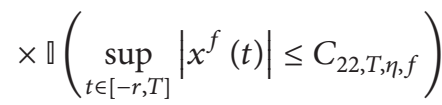

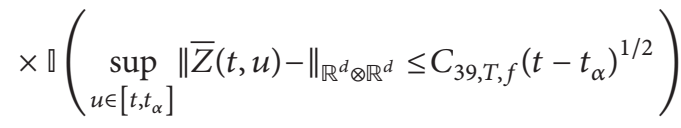

$$
\begin{aligned}
& =C_{41, T, \eta, f}\left(t-t_{\alpha}\right)^{d},
\end{aligned}
$$

which is strictly positive. Here, we will remark that there exists the constant $C_{41, T, \eta, f}>0$ with

$$
\frac{1}{2} \inf _{\zeta, t, g} \sum_{i=1}^{m}\left(\zeta \cdot A_{i}(t, g)\right)^{2}-C_{40, T, \eta, f}\left(t-t_{\alpha}\right) \geq C_{41, T, \eta, f}
$$

because the functions $A_{i}(i=1, \ldots, m)$ satisfy the uniformly elliptic condition (24), and $t_{\alpha}$ is sufficiently close to $t$, which justifies the sixth inequality.

For $f \in H$, let $\left\{X^{\varepsilon, f}(t) ; t \in[-r, T]\right\}$ be the $\mathbb{R}^{d}$-valued process determined by the following equation:

$$
\begin{aligned}
X^{\varepsilon, f}(t)= & \eta(t) \quad(t \in[-r, 0]), \\
d X^{\varepsilon, f}(t)= & A_{0}\left(t, X_{t}^{\varepsilon, f}\right) d t+\varepsilon A\left(t, X_{t}^{\varepsilon, f}\right) d W(t) \\
& +A\left(t, X_{t}^{\varepsilon, f}\right) \dot{f}(t) d t \quad(t \in(0, T]) .
\end{aligned}
$$

Let $\left\{\widetilde{Z}^{f}(t) ; t \in[-r, T]\right\}$ be the $\mathbb{R}^{d}$-valued process determined by the following equation:

$$
\begin{aligned}
\widetilde{Z}^{f}(t)= & 0 \quad(t \in[-r, 0]) \\
d \widetilde{Z}^{f}(t)= & A\left(t, x_{t}^{f}\right) d W(t)+\nabla A_{0}\left(t, x_{t}^{f}\right) \widetilde{Z}_{t}^{f} d t \\
& +\nabla A\left(t, x_{t}^{f}\right) \widetilde{Z}_{t}^{f} \dot{f}(t) d t \quad(t \in(0, T])
\end{aligned}
$$

Lemma 19. Let $t \in(0, T]$. It holds that

$$
\lim _{\varepsilon \searrow 0}\left\|Y^{\varepsilon, f}(t)-\widetilde{Z}^{f}(t)\right\|_{k, p}=0,
$$

for any $p>1$ and $k \in \mathbb{Z}_{+}$, where $Y^{\varepsilon, f}(t)=\left(X^{\varepsilon, f}(t)-x^{f}(t)\right) / \varepsilon$. Proof. We will prove the statement along the following procedure.

Step 1 . For any $p>1$,

$$
\lim _{\varepsilon \searrow 0} \mathbb{E}\left[\sup _{t \in[-r, T]}\left|X^{\varepsilon, f}(t)-x^{f}(t)\right|^{p}\right]=0 .
$$

In fact, since

$$
\begin{aligned}
X^{\varepsilon, f}(t)-x^{f}(t)= & \int_{0}^{t}\left\{A_{0}\left(s, X_{s}^{\varepsilon, f}\right)-A_{0}\left(s, x_{s}^{f}\right)\right\} d s \\
& +\int_{0}^{t}\left\{A\left(s, X_{s}^{\varepsilon, f}\right)-A\left(s, x_{s}^{f}\right)\right\} \dot{f}(s) d s \\
& +\varepsilon \int_{0}^{t} A\left(s, X_{s}^{\varepsilon, f}\right) d W(s),
\end{aligned}
$$

for $t \in[0, T]$, and the coefficients $A_{i}(i=0,1, \ldots, m)$ satisfy the Lipschitz condition and the linear growth condition, we 
can get the assertion of Step 1 by using the Hölder inequality, the Burkholder inequality, and the Gronwall inequality.

Step 2. For any $p>1$,

$$
\lim _{\varepsilon \searrow 0} \mathbb{E}\left[\sup _{t \in[-r, T]}\left|Y^{\varepsilon, f}(t)-\widetilde{Z}^{f}(t)\right|^{p}\right]=0,
$$

which tells us to see that the assertion of Lemma 19 holds in the case of $k=0$.

In fact, we will remark that

$$
\begin{aligned}
\frac{A_{i}\left(s, X_{s}^{\varepsilon, f}\right)-A_{i}\left(s, x_{s}^{f}\right)}{\varepsilon}-\nabla A_{i}\left(s, x_{s}^{f}\right) \widetilde{Z}_{s}^{f} \\
=\nabla A_{i}\left(s, x_{s}^{f}\right)\left(Y_{s}^{\varepsilon, f}-\widetilde{Z}_{s}^{f}\right) \\
\quad+\frac{1}{2} \nabla^{2} A_{i}\left(s, \sigma X_{s}^{\varepsilon, f}+(1-\sigma) x_{s}^{f}\right)\left[X_{s}^{\varepsilon, f}-x_{s}^{f}, Y_{s}^{\varepsilon, f}\right],
\end{aligned}
$$

from the Taylor theorem for $i=0,1, \ldots, m$, where $0<\sigma<1$ is the constant. Here, for each $t \in[0, T]$ and $\varphi \in C([-r, 0]$; $\left.\mathbb{R}^{d}\right), \nabla^{2} A_{i}(t, \varphi)[\cdot, \cdot]$ is the bilinear mapping on $C([-r, 0]$; $\left.\mathbb{R}^{d}\right) \times C\left([-r, 0] ; \mathbb{R}^{d}\right)$. Since

$$
\begin{aligned}
Y^{\varepsilon, f}(t) & -\widetilde{Z}^{f}(t) \\
= & \int_{0}^{t}\left[\frac{A_{0}\left(s, X_{s}^{\varepsilon, f}\right)-A_{0}\left(s, x_{s}^{f}\right)}{\varepsilon}-\nabla A_{0}\left(s, x_{s}^{f}\right) \widetilde{Z}_{s}^{f}\right] d s \\
& +\int_{0}^{t}\left[\frac{A\left(s, X_{s}^{\varepsilon, f}\right)-A\left(s, x_{s}^{f}\right)}{\varepsilon}-\nabla A\left(s, x_{s}^{f}\right) \widetilde{Z}_{s}^{f}\right] \dot{f}(s) d s \\
& +\int_{0}^{t}\left\{A\left(s, X_{s}^{\varepsilon, f}\right)-A\left(s, x_{s}^{f}\right)\right\} d W(s),
\end{aligned}
$$

for $t \in[0, T]$, and the coefficients $A_{i}(t, \cdot)(i=0,1, \ldots, m)$ are in $C_{1+, b}^{\infty}\left(C\left([-r, 0] ; \mathbb{R}^{d}\right) ; \mathbb{R}^{d}\right)$ with respect to the second variable in $C\left([-r, 0] ; \mathbb{R}^{d}\right)$ for each $t \in[0, T]$, we can get the assertion in Step 2 via the Hölder inequality, the Burkholder inequality, and the Gronwall inequality.

Step 3. Let $u \in[0, T]$. Then, for any $p>1$,

$$
\sup _{0<\varepsilon \leq 1} \mathbb{E}\left[\sup _{t \in[-r, T]}\left|D_{u} Y^{\varepsilon, f}(t)\right|^{p}\right]<+\infty
$$

Remark that

$$
\begin{aligned}
& D_{u} Y^{\varepsilon, f}(t) \\
& \quad=\int_{0}^{u \wedge t}\left\{A\left(s, X_{s}^{\varepsilon, f}\right)+\frac{A\left(s, X_{s}^{\varepsilon, f}\right)-A\left(s, x_{s}^{f}\right)}{\varepsilon}\right\} d s \\
& \quad+\int_{0}^{t} \frac{1}{\varepsilon}\left\{\nabla A_{0}\left(s, X_{s}^{\varepsilon, f}\right) D_{u} X_{s}^{\varepsilon, f}-\nabla A_{0}\left(s, x_{s}^{f}\right) D_{u} x_{s}^{f}\right\} d s \\
& \quad+\int_{0}^{t} \frac{1}{\varepsilon}\left\{\nabla A\left(s, X_{s}^{\varepsilon, f}\right) D_{u} X_{s}^{\varepsilon, f}-\nabla A\left(s, x_{s}^{f}\right) D_{u} x_{s}^{f}\right\} \dot{f}(s) d s \\
& \quad+\int_{0}^{t} \nabla A\left(s, X_{s}^{\varepsilon, f}\right) D_{u} X_{s}^{\varepsilon, f} d W(s),
\end{aligned}
$$

for $t \in[u, T]$. Since

$$
\begin{aligned}
D_{u} x^{f}(t)= & \int_{0}^{u \wedge t} A\left(s, x_{s}^{f}\right) d s+\int_{0}^{t} \nabla A_{0}\left(s, x_{s}^{f}\right) D_{u} x_{s}^{f} d s \\
& +\int_{0}^{t} \nabla A\left(s, x_{s}^{f}\right) D_{u} x_{s}^{f} \dot{f}(s) d s,
\end{aligned}
$$

as seen in Proposition 18, we have

$$
\sup _{t \in[-r, T]}\left|D_{u} x^{f}(t)\right| \leq C_{42, T, \eta, f}
$$

Moreover, similarly to Proposition 5, we have

$$
\mathbb{E}\left[\sup _{t \in[-r, T]}\left|D_{u} X^{\varepsilon, f}(t)\right|^{p}\right] \leq C_{43, p, T, \eta, f},
$$

for any $p>1$. Then, the assertion in Step 3 can be justified by using the Hölder inequality, the Burkholder inequality, and the Gronwall inequality.

Step 4 . Let $u \in[0, T]$. Then, for any $p>1$,

$$
\lim _{\varepsilon \downarrow 0} \mathbb{E}\left[\sup _{t \in[-r, T]}\left|D_{u} Y^{\varepsilon, f}(t)-D_{u} \widetilde{Z}^{f}(t)\right|^{p}\right]=0 .
$$


In fact, since

$$
\begin{aligned}
& D_{u} Y^{\varepsilon, f}(t) \\
& =\int_{0}^{u}\left\{A\left(s, X_{s}^{\varepsilon, f}\right)+\frac{A\left(s, X_{s}^{\varepsilon, f}\right)-A\left(s, x_{s}^{f}\right)}{\varepsilon}\right\} \rrbracket_{(s \leq t)} d s \\
& +\int_{0}^{t} \frac{1}{\varepsilon}\left\{\nabla A_{0}\left(s, X_{s}^{\varepsilon, f}\right) D_{u} X_{s}^{\varepsilon, f}-\nabla A_{0}\left(s, x_{s}^{f}\right) D_{u} x_{s}^{f}\right\} d s \\
& +\int_{0}^{t} \frac{1}{\varepsilon}\left\{\nabla A\left(s, X_{s}^{\varepsilon, f}\right) D_{u} X_{s}^{\varepsilon, f}\right. \\
& \left.-\nabla A\left(s, x_{s}^{f}\right) D_{u} x_{s}^{f}\right\} \dot{f}(s) d s \\
& +\int_{0}^{t} \nabla A\left(s, X_{s}^{\varepsilon, f}\right) D_{u} X_{s}^{\varepsilon, f} d W(s), \\
& D_{u} \widetilde{Z}^{f}(t) \\
& =\int_{0}^{u}\left\{A\left(s, x_{s}^{f}\right)+\nabla A\left(s, x_{s}^{f}\right) \widetilde{Z}_{s}^{f}\right\} \rrbracket_{(s \leq t)} d s \\
& +\int_{0}^{t} \nabla A_{0}\left(s, x_{s}^{f}\right) D_{u} \widetilde{Z}_{s}^{f} d s \\
& +\int_{0}^{t} \nabla^{2} A_{0}\left(s, x_{s}^{f}\right)\left[D_{u} x_{s}^{f}, \widetilde{Z}_{s}^{f}\right] d s \\
& +\int_{0}^{t} \nabla A\left(s, x_{s}^{f}\right) D_{u} \widetilde{Z}_{s}^{f} \dot{f}(s) d s \\
& +\int_{0}^{t} \nabla^{2} A\left(s, x_{s}^{f}\right)\left[D_{u} x_{s}^{f}, \widetilde{Z}_{s}^{f}\right] \dot{f}(s) d s \\
& +\int_{0}^{t} \nabla A\left(s, x_{s}^{f}\right) D_{u} x_{s}^{f} d W(s),
\end{aligned}
$$

for $t \in[u, T]$, and the coefficients $A_{i}(t, \cdot)(i=0,1, \ldots, m)$ are in $C_{1+, b}^{\infty}\left(C\left([-r, 0] ; \mathbb{R}^{d}\right) ; \mathbb{R}^{d}\right)$ with respect to the second variable in $C\left([-r, 0] ; \mathbb{R}^{d}\right)$ for each $t \in[0, T]$, the assertion can be obtained via the Hölder inequality, the Burkholder inequality, and the Gronwall inequality. Here $\nabla^{2} A\left(s, x_{s}^{f}\right)=$ $\left(\nabla^{2} A_{1}\left(s, x_{s}^{f}\right), \ldots, \nabla^{2} A_{m}\left(s, x_{s}^{f}\right)\right)$.

Step 5 . Let $k \in \mathbb{N}$ be arbitrary, and $u_{1}, \ldots, u_{k} \in[0, T]$. Then, for any $p>1$,

$$
\lim _{\varepsilon \searrow 0} \mathbb{E}\left[\sup _{t \in[-r, T]}\left|D_{u_{1}, \ldots, u_{k}}^{k} Y^{\varepsilon, f}(t)-D_{u_{1}, \ldots, u_{k}}^{k} \widetilde{Z}^{f}(t)\right|^{p}\right]=0 .
$$

We have already proved the case of $k=1$ in Step 4. Remark that

$$
\begin{aligned}
D_{u_{1}, \ldots, u_{k}}^{k} & \left(\int_{0}^{t} \varphi(s) d W(s)\right) \\
= & \int_{0}^{t} D_{u_{1}, \ldots, u_{k}}^{k}(\varphi(s)) d W(s)
\end{aligned}
$$

$$
\begin{array}{r}
+\sum_{j=1}^{k} D_{u_{1}, \ldots, u_{j-1}, u_{j+1}, \ldots, u_{k}}^{k-1}\left(\int_{0}^{u_{j} \wedge t} \varphi(s) d s\right), \\
D_{u_{1}, \ldots, u_{k}}^{k}\left(\int_{0}^{t} \psi(s) d s\right)=\int_{0}^{t} D_{u_{1}, \ldots, u_{k}}^{k}(\psi(s)) d s,
\end{array}
$$

for adapted processes $\varphi$ and $\psi$ with nice properties. Then, we can get the assertion by induction on $k \in \mathbb{N}$.

Then, the assertion is the direct consequences of Step 2 and Step 5. The proof of Lemma 19 is complete.

Theorem 20 (Lower estimate). Suppose that the $\mathbb{R}^{d}$-valued functions $A_{i}(i=1, \ldots, m)$ satisfy the uniformly elliptic condition (24). Then, it holds that

$$
\liminf _{\varepsilon \searrow 0} \varepsilon^{2} \ln p^{\varepsilon}(t, y) \geq-\bar{I}(y),
$$

where the function $\bar{I}$ is given in Corollary 11.

Proof. Since the assertion of Theorem 20 is trivial in the case of $\bar{I}(y)=+\infty$, we will suppose that $\bar{I}(y)<+\infty$. Let $\Phi \in C_{0}^{\infty}\left(\mathbb{R}^{d} ; \mathbb{R}\right)$ be nonnegative. For sufficiently small $0<$ $\sigma<1$, recall the function $\Lambda_{\sigma}$ as introduced in the proof of Theorem 16: $\Lambda_{\sigma} \in C_{0}^{\infty}\left(\mathbb{R}^{d} ;[0,1]\right)$ such that

$$
\Lambda_{\sigma}(z)= \begin{cases}1 & (|z-y| \leq \sigma) \\ 0 & (|z-y|>2 \sigma)\end{cases}
$$

Then, the Girsanov theorem tells us to see that

$$
\begin{aligned}
\mathbb{E}\left[\Phi\left(X^{\varepsilon}(t)\right)\right] \\
=\mathbb{E}\left[\Phi\left(X^{\varepsilon, f}(t)\right) \exp \left(-\int_{0}^{t} \sum_{i=1}^{m} \frac{\dot{f}^{i}(s)}{\varepsilon} d W^{i}(s)-\frac{1}{2 \varepsilon^{2}}\|f\|_{H}^{2}\right)\right] \\
=\exp \left(-\frac{\|f\|_{H}^{2}+4 \sigma}{2 \varepsilon^{2}}\right) \\
\quad \times \mathbb{E}\left[\Phi\left(X^{\varepsilon, f}(t)\right) \exp \left(-\int_{0}^{t} \sum_{i=1}^{m} \frac{\dot{f}^{i}(s)}{\varepsilon} d W^{i}(s)+\frac{2 \sigma}{\varepsilon^{2}}\right)\right] \\
\geq \exp \left(-\frac{\|f\|_{H}^{2}+4 \sigma}{2 \varepsilon^{2}}\right) \\
\quad \times \mathbb{E}\left[\Phi\left(X^{\varepsilon, f}(t)\right) \square\left(\varepsilon \int_{0}^{t} \sum_{i=1}^{m} \dot{f}^{i}(s) d W^{i}(s) \leq 2 \sigma\right)\right] \\
\geq \exp \left(-\frac{\|f\|_{H}^{2}+4 \sigma}{2 \varepsilon^{2}}\right) \\
\quad \times \mathbb{E}\left[\Phi\left(X^{\varepsilon, f}(t)\right) \Lambda_{\sigma}\left(\varepsilon \int_{0}^{t} \sum_{i=1}^{m} \dot{f}^{i}(s) d W^{i}(s)\right)\right] .
\end{aligned}
$$


Here, the third inequality comes from the nonnegativity in the exponent

$$
-\frac{1}{\varepsilon} \int_{0}^{t} \dot{f}(s) d W(s)+\frac{2 \sigma}{\varepsilon^{2}} \geq 0,
$$

while the forth inequality holds because of $0 \leq \Lambda_{\sigma} \leq 1$ and $\Lambda_{\sigma} \neq 0$ on the complement of $[-2 \sigma, 2 \sigma]$. Thus, the limiting argument enables us to see that

$$
\begin{aligned}
p^{\varepsilon}(t, y) \geq & \exp \left(-\frac{\|f\|_{H}^{2}+4 \sigma}{2 \varepsilon^{2}}\right) \\
& \times \mathbb{E}\left[\delta_{y}\left(X^{\varepsilon, f}(t)\right) \Lambda_{\sigma}\left(\varepsilon \int_{0}^{t} \sum_{i=1}^{m} \dot{f}^{i}(s) d W^{i}(s)\right)\right] \\
= & \varepsilon^{-d} \exp \left(-\frac{\|f\|_{H}^{2}+4 \sigma}{2 \varepsilon^{2}}\right) \\
& \times \mathbb{E}\left[\delta_{0}\left(Y^{\varepsilon, f}(t)\right) \Lambda_{\sigma}\left(\varepsilon \int_{0}^{t} \sum_{i=1}^{m} \dot{f}^{i}(s) d W^{i}(s)\right)\right],
\end{aligned}
$$

where $\delta_{y}$ is the Dirac delta function. Since

$$
\lim _{\varepsilon \searrow 0} \mathbb{E}\left[\delta_{0}\left(Y^{\varepsilon, f}(t)\right) \Lambda_{\sigma}\left(\varepsilon \int_{0}^{t} \sum_{i=1}^{m} \dot{f}^{i}(s) d W^{i}(s)\right)\right] \leq 1,
$$

from Lemma 19, we have

$$
\begin{gathered}
\lim _{\varepsilon \unlhd 0} \varepsilon^{2} \ln \left(\varepsilon^{-d} \mathbb{E}\left[\delta_{0}\left(Y^{\varepsilon, f}(t)\right) \Lambda_{\sigma}\left(\varepsilon \int_{0}^{t} \sum_{i=1}^{m} \dot{f}^{i}(s) d W^{i}(s)\right)\right]\right) \\
=0 .
\end{gathered}
$$

Moreover, from the definition of the function $\bar{I}(y)$, we can find $f \in H$ with $y=x^{f}(t)$ such that

$$
\frac{\|f\|_{H}^{2}}{2} \leq \bar{I}(y)+\sigma \text {. }
$$

Hence, it holds that

$$
\liminf _{\varepsilon \downarrow 0} \varepsilon^{2} \ln p^{\varepsilon}(t, y) \geq-\left(\frac{\|f\|_{H}^{2}}{2}+2 \sigma\right) \geq-\bar{I}(y)-3 \sigma .
$$

Taking the limit as $\sigma \searrow 0$ completes the proof.

Corollary 21. Suppose that the $\mathbb{R}^{d}$-valued functions $A_{i}(i=$ $1, \ldots, m)$ satisfy the uniformly elliptic condition (24). Then, it holds that

$$
p^{\varepsilon}(t, y) \sim \exp \left[-\frac{\bar{I}(y)}{\varepsilon^{2}}\right]
$$

as $\varepsilon \searrow 0$, where the function $\bar{I}$ is given in Corollary 11 .
Proof. Direct consequences of Theorems 16 and 20.

Finally, we will study the asymptotic behavior of the density $p(t, y)$ for $X(t)$ in a short time. Let $0<r_{0} \leq r$ be a constant, and $x \in \mathbb{R}^{d}$. We will consider the case

$$
\begin{gathered}
\eta(t)=x \quad(t \in[-r, 0]), \\
A_{0}(t, f) \equiv 0, \quad A_{i}(t, f)=\widetilde{A}_{i}(\widetilde{f}, f(0)) \quad(i=1, \ldots, m),
\end{gathered}
$$

where $\tilde{f} \in C\left([-r, 0] ; \mathbb{R}^{d}\right)$ such that $\tilde{f}(t)=f(t)(t \in[-r$, $\left.\left.-r_{0}\right]\right)$, and $\widetilde{f}(t)=\widetilde{f}\left(-r_{0}\right)\left(t \in\left[-r_{0}, 0\right]\right)$, for $f \in C([-r$, $\left.0] ; \mathbb{R}^{d}\right)$. Suppose that the functions $\widetilde{A}_{i}(i=1, \ldots, m)$ satisfy the uniformly elliptic condition of the form:

$$
\inf _{\zeta \in \mathbb{S}^{d-1}} \inf _{f \in C\left(\left[-r,-r_{0}\right] ; \mathbb{R}^{d}\right)} \inf _{y \in \mathbb{R}^{d}} \sum_{i=1}^{m}\left(\zeta \cdot \widetilde{A}_{i}(f, y)\right)^{2}>0 .
$$

For $0<\varepsilon \leq 1$, let $X=\{X(t) ; t \in[-r, T]\}$, and let $X^{\varepsilon}=$ $\left\{X^{\varepsilon}(t) ; t \in[-r, T]\right\}$ be the $\mathbb{R}^{d}$-valued processes determined by the equations of the form:

$$
\begin{gathered}
X(t)=x \quad(t \in[-r, 0]), \\
d X(t)=\widetilde{A}\left(\widetilde{X}_{t}, X(t)\right) d W(t) \quad(t \in(0, T]), \\
X^{\varepsilon}(t)=x \quad(t \in[-r, 0]), \\
d X^{\varepsilon}(t)=\varepsilon \widetilde{A}\left(\widetilde{X}_{t}^{\varepsilon}, X^{\varepsilon}(t)\right) d W(t) \quad(t \in(0, T]),
\end{gathered}
$$

where $\widetilde{A}=\left(\widetilde{A}_{1}, \ldots, \widetilde{A}_{m}\right)$. Remark that $X=\left.X^{\varepsilon}\right|_{\varepsilon=1}$. Denote by $p(t, y)$ (or, $p^{\varepsilon}(t, y)$ ) the density for the probability law of $X(t)\left(X^{\varepsilon}(t)\right.$, resp.), whose existence can be justified under the uniformly elliptic condition (122) on the coefficients $\widetilde{A}_{i}(i=$ $1, \ldots, m)$. Then, we have the following.

Corollary 22. Suppose that the functions $\widetilde{A}_{i}(i=1, \ldots, m)$ satisfy the uniformly elliptic condition (122). Then, it holds that

$$
p(t, y) \sim \exp \left[-\frac{r_{0} \bar{I}(y)}{t}\right] \quad(t \searrow 0) .
$$

Proof. Recall that

$$
\begin{aligned}
X\left(\varepsilon^{2} r_{0}\right) & =x+\int_{0}^{\varepsilon^{2} r_{0}} \widetilde{A}\left(\widetilde{X}_{s}, X(s)\right) d W(s) \\
& =x+\varepsilon \int_{0}^{r_{0}} \widetilde{A}\left(\widetilde{X}_{\varepsilon^{2} s}, X\left(\varepsilon^{2} s\right)\right) d W(s) \\
& =x+\varepsilon \int_{0}^{r_{0}} \widetilde{A}\left(x i d, X\left(\varepsilon^{2} s\right)\right) d W(s),
\end{aligned}
$$

where id $\in C\left(\left[-r,-r_{0}\right] ; \mathbb{R}^{d}\right)$ such that $i d(t)=1(t \in[-r$, $\left.\left.-r_{0}\right]\right)$. Here, the second equality holds from the scaling property on the Brownian motion $W$, while the third equality follows from $\varepsilon^{2} s-r_{0} \leq 0$. On the other hand, recall that

$$
\begin{aligned}
X^{\varepsilon}\left(r_{0}\right) & =x+\varepsilon \int_{0}^{r_{0}} \widetilde{A}\left(\widetilde{X}_{s}^{\varepsilon}, X^{\varepsilon}(s)\right) d W(s) \\
& =x+\varepsilon \int_{0}^{r_{0}} \widetilde{A}\left(x i d, X^{\varepsilon}(s)\right) d W(s),
\end{aligned}
$$


because of $s-r_{0} \leq 0$. From the uniqueness of the solutions, we have $X\left(\varepsilon^{2} r_{0}\right)=X^{\varepsilon}\left(r_{0}\right)$ in the sense of the probability law. Hence, we can get

$$
p\left(\varepsilon^{2} r_{0}, y\right)=p^{\varepsilon}\left(r_{0}, y\right)
$$

As for the density $p^{\varepsilon}\left(r_{0}, y\right)$, we have already obtained the asymptotic behavior of the form:

$$
p^{\varepsilon}\left(r_{0}, y\right) \sim \exp \left[-\frac{\bar{I}(y)}{\varepsilon^{2}}\right],
$$

as $\varepsilon \searrow 0$, in Corollary 21. Taking $t=\varepsilon^{2} r_{0}$ completes the proof.

Remark 23. In particular, consider the case of

$$
\begin{gathered}
A_{0}(s, f)=0, \quad A_{i}(s, f)=\bar{A}_{i}(f(0)) \quad(i=1, \ldots, m), \\
\eta(t)=x \quad(t \in[-r, 0]),
\end{gathered}
$$

where $\bar{A}_{i} \in C_{1+, b}^{\infty}\left(\mathbb{R}^{d} ; \mathbb{R}^{d}\right)$ such that the functions $\bar{A}_{i}(i=$ $1, \ldots, m)$ satisfy the uniformly elliptic condition of the form:

$$
\inf _{\zeta \in \mathbb{S}^{d-1}} \inf _{y \in \mathbb{R}^{d}} \sum_{i=1}^{m}\left(\zeta \cdot \bar{A}_{i}(y)\right)^{2}>0 .
$$

Then, our equation can be written as follows:

$$
\begin{gathered}
X(t)=x \quad(t \in[-r, 0]), \\
d X(t)=\bar{A}(X(t)) d W(t) \quad(t \in(0, T]),
\end{gathered}
$$

where $\bar{A}=\left(\bar{A}_{1}, \ldots, \bar{A}_{m}\right)$. Although our settings include the effect of the time-delay parameter $r$, the effect of the parameter $r$ in (131) can be ignored. Hence, the solution $\{X(t) ; t \in[-r, T]\}$ is the diffusion process, so we have only to choose $r=1$ in the starting point of our study. Moreover, the choice of $r=1$ tells us to see that Corollary 22 is the well-known fact, that is, the Varadhan-type estimate, on the asymptotic behavior of the density function for diffusion processes. Hence, Corollary 22 can be also regarded as the generalization of the short-time estimate of the density for diffusion processes.

Remark 24. Ferrante et al. in [10] discussed the large deviation principle for the solution process $X^{\varepsilon}$ and the asymptotic estimate of the density, in the case of

$$
A_{i}(s, f)=\widetilde{A}_{i}(s, f(s-r)) \quad(i=1, \ldots, m),
$$

where $\widetilde{A}_{i}:[0, T] \times \mathbb{R}^{d} \rightarrow \mathbb{R}^{d}$ with $\widetilde{A}_{i}(t, \cdot) \in C_{b}^{\infty}\left(\mathbb{R}^{d} ; \mathbb{R}^{d}\right)$ for each $t \in[0, T]$. Moreover, suppose that the functions $\widetilde{A}_{i}(i=$ $1, \ldots, m)$ satisfy the uniformly elliptic condition of the form:

$$
\inf _{\zeta \in \mathbb{S}^{d-1}} \inf _{t \in[0, T]} \inf _{y \in \mathbb{R}^{d}} \sum_{i=1}^{m}\left(\zeta \cdot A_{i}(t, y)\right)^{2}>0 .
$$

On the other hand, Mohammed and Zhang in [11] studied the large deviation principle for the solution process $X^{\varepsilon}$, in the case of

$$
A_{i}(t, f)=\widetilde{A}_{i}(t, f(t-r), f(t)) \quad(i=1, \ldots, m),
$$

where $\widetilde{A}_{i}:[0, T] \times \mathbb{R}^{d} \times \mathbb{R}^{d} \rightarrow \mathbb{R}^{d}$ with $\widetilde{A}_{i}(t, \cdot, \cdot) \in$ $C_{1+, b}^{\infty}\left(\mathbb{R}^{d} \times \mathbb{R}^{d} ; \mathbb{R}^{d}\right)$.

Since the special forms of the coefficients on the diffusion terms are quite essential in their arguments [10,11], our situation cannot be included in their frameworks at all.

\section{Acknowledgments}

The authors are grateful to an anonymous referee for valuable comments and suggestions. This work is partially supported by Ministry of Education, Culture, Sports, Science, and Technology and Grant-in-Aid for Encouragement of Young Scientists, 23740083. This work is also partially supported from the Research Council of Norway, 219005/F11. This work was largely carried out while the second author stayed at the Center of Mathematics for Applications, University of Oslo, from August to September, 2012, on his leave from Osaka City University.

\section{References}

[1] K. Itô and M. Nisio, "On stationary solutions of a stochastic differential equation," Journal of Mathematics of Kyoto University, vol. 4, pp. 1-75, 1964.

[2] S. E. A. Mohammed, Stochastic Functional Differential Equations, Pitman, Boston, Mass, USA, 1984.

[3] J.-M. Bismut, Large Deviations and the Malliavin Calculus, Birkhäuser, 1984.

[4] S. Kusuoka and D. Stroock, "Applications of the Malliavin calculus. I," in Stochastic Analysis (Katata/Kyoto, 1982), pp. 271306, 1984.

[5] R. Léandre, "Majoration en temps petit de la densité d'une diffusion dégénérée," Probability Theory and Related Fields, vol. 74, no. 2, pp. 289-294, 1987.

[6] R. Léandre, "Minoration en temps petit de la densité d'une diffusion dégénérée," Journal of Functional Analysis, vol. 74, no. 2, pp. 399-414, 1987.

[7] R. Léandre, "Intégration dans la fibre associée à une diffusion dégénérée," Probability Theory and Related Fields, vol. 76, no. 3, pp. 341-358, 1987.

[8] R. Léandre, "A simple proof for a large deviation theorem," in Barcelona Seminar on Stochastic Analysis (St. Feliu de Guíxols, 1991), pp. 72-76, 1993.

[9] D. Nualart, "Analysis on Wiener space and anticipating stochastic calculus," in Ecole d'été de Probabilités de Saint-Flour 1995, vol. 1690 of Lecture Notes in Mathematics, pp. 123-227, Springer, Berlin, Germany, 1998.

[10] M. Ferrante, C. Rovira, and M. Sanz-Solé, "Stochastic delay equations with hereditary drift: estimates of the density," Journal of Functional Analysis, vol. 177, no. 1, pp. 138-177, 2000.

[11] S. E. A. Mohammed and T. Zhang, "Large deviations for stochastic systems with memory," Discrete and Continuous Dynamical Systems, vol. 6, no. 4, pp. 881-893, 2006. 
[12] S. E. A. Mohammed, "Stochastic differential systems with memory: theory, examples and applications," in Stochastic Analysis and Related Topics VI (Geilo, 1996), vol. 42 of Progress in Probability, pp. 1-77, Birkhäuser, Boston, Mass, USA, 1998.

[13] G. Di Nunno, B. Øksendal, and F. Proske, Malliavin Calculus for Lévy Processes with Applications to Finance, Springer, 2009.

[14] D. Nualart, The Malliavin Calculus and Related Topics, Springer, 2nd edition, 2006.

[15] T. Komatsu and A. Takeuchi, "Simplified probabilistic approach to the Hörmander theorem," Osaka Journal of Mathematics, vol. 38, no. 3, pp. 681-691, 2001.

[16] A. Takeuchi, "Malliavin calculus for degenerate stochastic functional differential equations," Acta Applicandae Mathematicae, vol. 97, no. 1-3, pp. 281-295, 2007.

[17] A. Dembo and O. Zeitouni, Large Deviations Techniques and Applications, Springer, 2nd edition, 1998.

[18] R. Azencott, "Grandes déviations et applications," in Ecole d'été de Probabilites de Saint-Flour VIII (1978), vol. 774 of Lecture Notes in Mathematics, pp. 1-176, Springer, Berlin, Germany, 1980. 


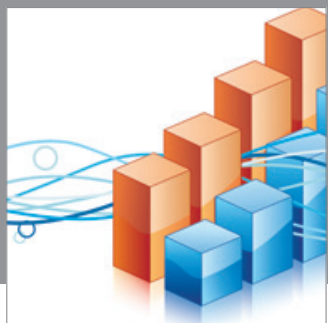

Advances in

Operations Research

mansans

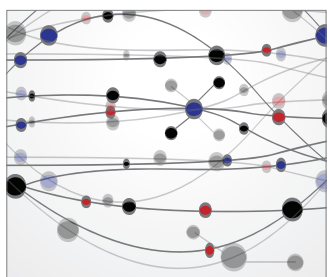

The Scientific World Journal
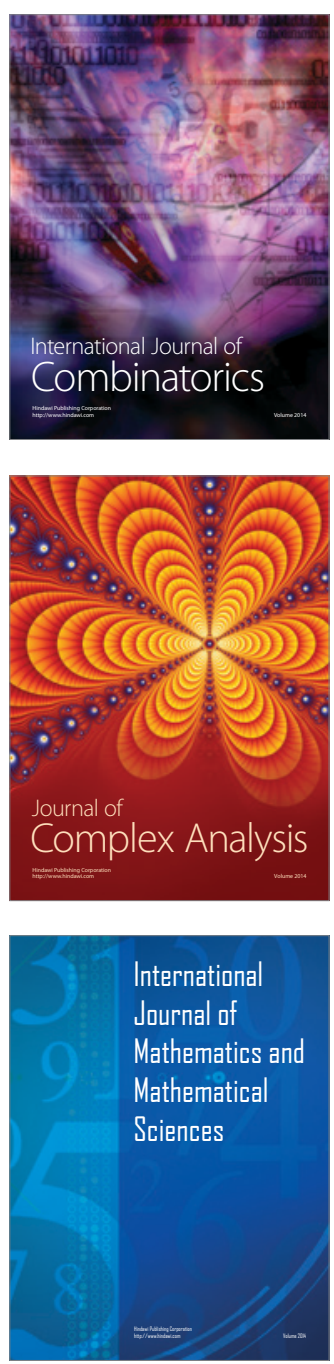
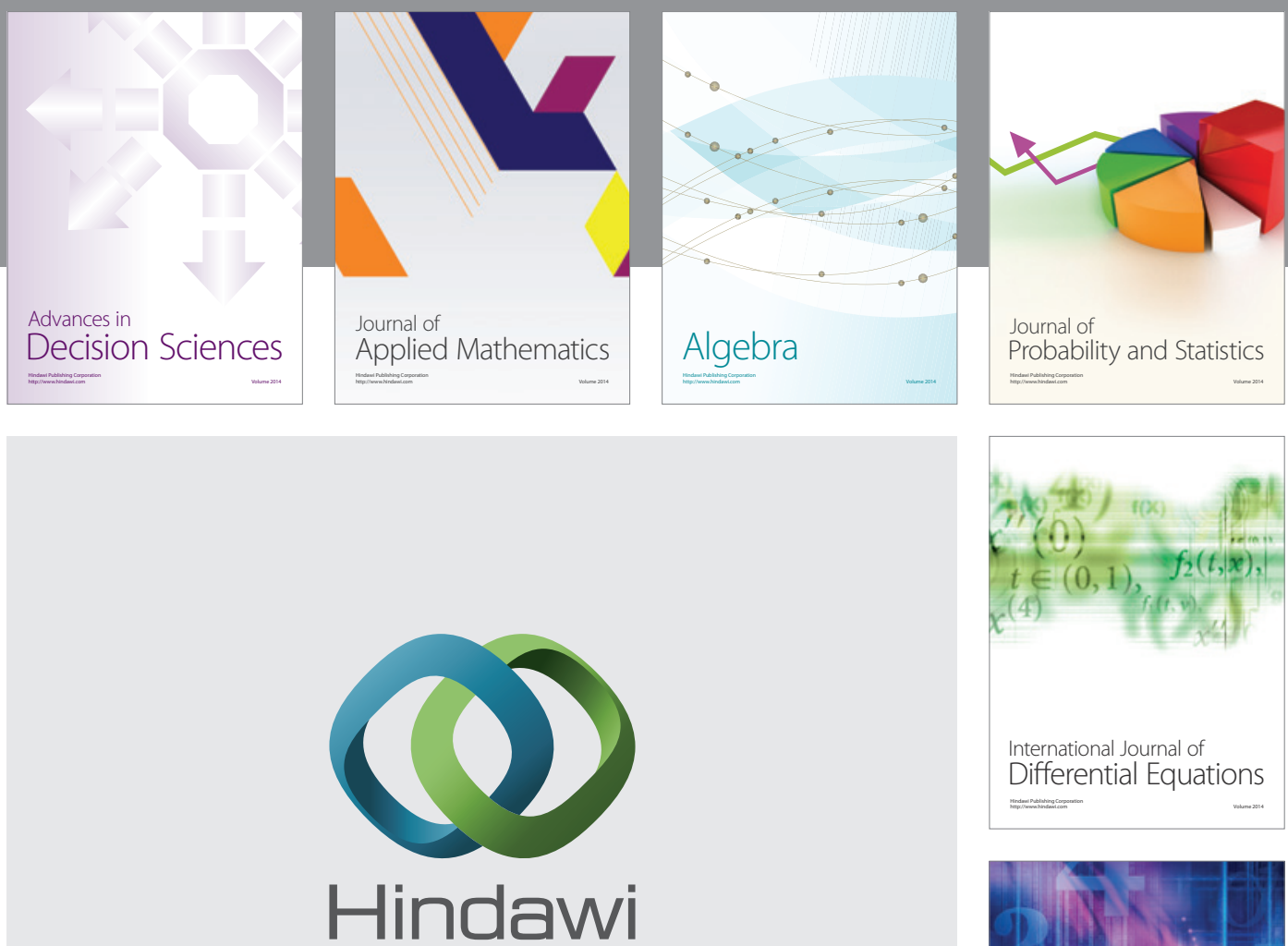

Submit your manuscripts at http://www.hindawi.com
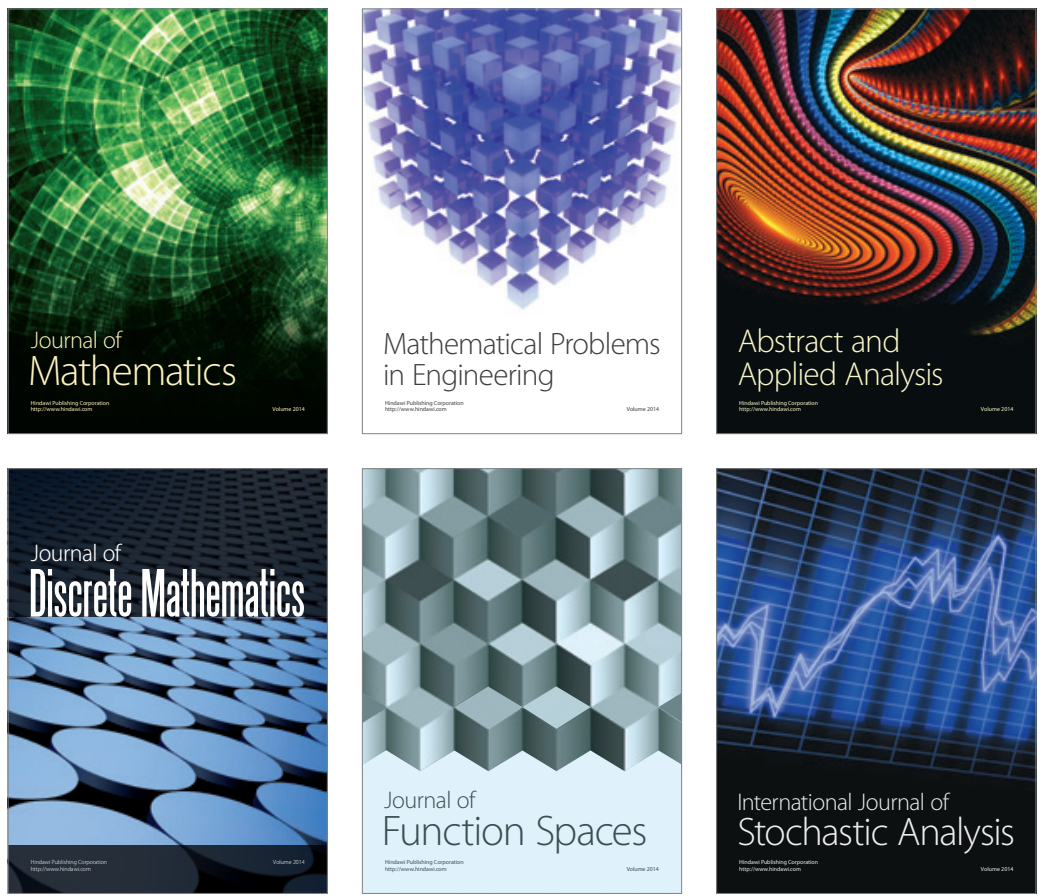

Journal of

Function Spaces

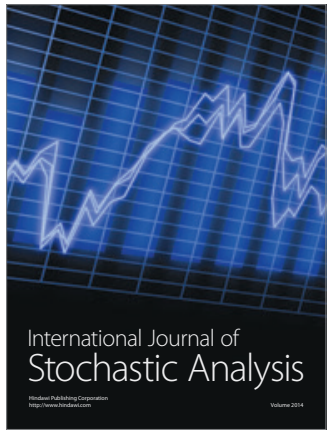

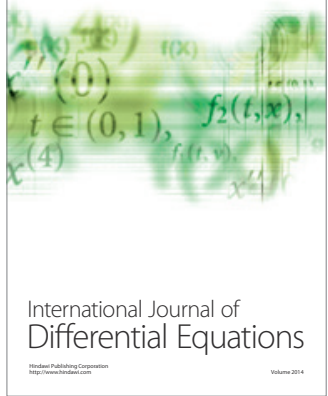
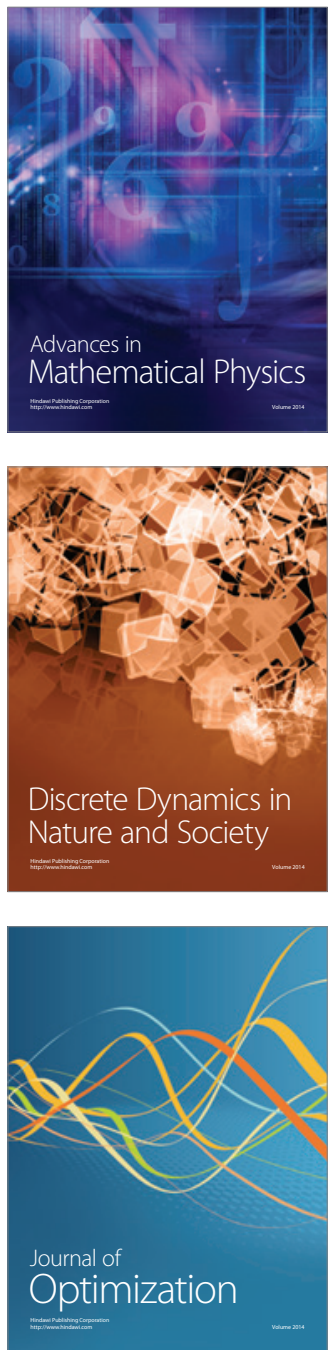